\title{
RECQ1 expression is upregulated in response to DNA damage and in a p53-dependent manner
}

\author{
Swetha Parvathaneni ${ }^{1, *}$, Xing Lu ${ }^{1, *}$, Ritu Chaudhary ${ }^{2}$, Ashish Lal ${ }^{2}$, Srinivasan \\ Madhusudan ${ }^{3}$ and Sudha Sharma ${ }^{1}$ \\ ${ }^{1}$ Department of Biochemistry and Molecular Biology, College of Medicine, Howard University, NW, Washington, DC, 20059, \\ USA \\ ${ }^{2}$ Regulatory RNAs and Cancer Section, Genetics Branch, National Cancer Institute, National Institutes of Health, Bethesda, \\ MD, 20892, USA \\ ${ }^{3}$ Academic Unit of Oncology, Division of Cancer and Stem Cells, School of Medicine, University of Nottingham, Nottingham, \\ NG51PB, UK \\ *These authors contributed equally to this work
}

Correspondence to: Sudha Sharma, email: sudha.sharma@howard.edu

Keywords: RecQ, helicase, DNA damage, p53, gene expression

Received: February 24, $2017 \quad$ Accepted: May 15, 2017

Published: May 27, 2017

Copyright: Parvathaneni et al. This is an open-access article distributed under the terms of the Creative Commons Attribution License 3.0 (CC BY 3.0), which permits unrestricted use, distribution, and reproduction in any medium, provided the original author and source are credited.

\section{ABSTRACT}

Sensitivity of cancer cells to DNA damaging chemotherapeutics is determined by DNA repair processes. Consequently, cancer cells may upregulate the expression of certain DNA repair genes as a mechanism to promote chemoresistance. Here, we report that RECQ1, a breast cancer susceptibility gene that encodes the most abundant RecQ helicase in humans, is a p53-regulated gene, potentially acting as a defense against DNA damaging agents. We show that RECQ1 mRNA and protein levels are upregulated upon treatment of cancer cells with a variety of DNA damaging agents including the DNA-alkylating agent methylmethanesulfonate (MMS). The MMSinduced upregulation of RECQ1 expression is p53-dependent as it was observed in p53-proficient but not in isogenic p53-deficient cells. The RECQ1 promoter is bound by endogenous p53 and is responsive to p53 in luciferase reporter assays suggesting that RECQ1 is a direct target of p53. Treatment with the chemotherapeutic drugs temozolomide and fotemustine also increased RECQ1 mRNA levels whereas depletion of RECQ1 enhanced cellular sensitivity to these agents. These results identify a previously unrecognized p53-mediated upregulation of RECQ1 expression in response to DNA damage and implicate RECQ1 in the repair of DNA lesions including those induced by alkylating and other chemotherapeutic agents.

\section{INTRODUCTION}

Genes encoding proteins that function in replication checkpoint, maintenance of stalled replication forks and homologous recombination repair are frequently upregulated in a variety of cancers [1-3]. The upregulation of these genes in highly proliferative cancer cells is believed to be a general adaptive response to chronic replication stress, activating replication origins to compensate for DNA replication fork stalling and inducing DNA repair to cope with chromosomal breakage. However, increase in the expression of regulatory proteins of DNA damage-sensing and repair pathways contribute to improved DNA repair capacity, increased DNA damage tolerance, and the failure of cell death pathways. Consequently, upregulation of DNA repair genes is a mechanism to promote chemoresistance in tumor cells [3].

The RecQ helicases, described as caretakers of the genome, contribute multiple catalytic activities to DNA replication, repair, transcription, and telomere maintenance [4-6]. In humans, functional defects in RecQ proteins predispose to cancer. At the cellular level, loss of their functions leads to increased DNA damage, genomic instability and enhanced sensitivity to a variety 
of chemotherapeutic agents. The tumor suppressor roles of RecQ proteins are mediated through their genome caretaker roles; and the evidence that RecQ proteins directly regulate tumorigenesis is scarce. The elevated expression of RecQ helicases in rapidly proliferating cells and in many human cancers suggests possible roles in resistance to DNAdamaging agents and their expression levels may predict the responsiveness of cancers to certain chemotherapeutic agents [4]. However, whether or not RecQ helicases are regulated at the mRNA and/or protein level to provide cellular protection against genotoxic stress is poorly known [7].

Here we wanted to investigate whether DNA damage modulates the expression of RECQ1, a RecQ family member critical for DNA repair mechanisms that restore productive replication following stress and prevent genomic instability [8]. RECQ1 (also known as RECQL or $R E C Q L 1)$ was recently identified as a breast cancer susceptibility gene [9, 10]. Previous studies have shown that $R E C Q 1$ is upregulated in rapidly dividing cells and its expression is higher in many cancer cell lines as compared to normal cells [11]. Furthermore, RECQ1 silencing reduces proliferation of cancer cells and suppresses tumor growth in mouse xenograft models $[12,13]$. RECQ1 can contribute to tumor development and progression by regulating the expression of key genes that promote cancer cell migration, invasion and metastasis $[14,15]$. Indeed, RECQ1 is frequently over-expressed and amplified in many cancer samples (http://www.cbioportal.org/publicportal); and altered $R E C Q 1$ expression is correlated with patient's response to therapy [16-20]. Consistent with this, suppression of RECQ1 expression in mice and human cells is manifested as constitutively elevated sister chromatid exchange, chromosomal breakage, and increased sensitivity to ionizing radiation $[21,22]$. RECQ1 is critical for telomere maintenance [23, 24], restores replication fork progression following stress [25-27], participates in DNA double strand break repair [28], responds to oxidative DNA damage [29, 30], and performs a mechanistic role in base excision repair (BER) pathway which removes chemical alterations to DNA bases such as oxidation and alkylation [31]. Thus, we hypothesized that overexpression of RECQ1 may provide a survival advantage to cancer cells by promoting the ability of cancer cells to tolerate genotoxic stress.

Herein, we demonstrate that RECQ1, the gene encoding the most abundant RecQ family protein in humans, is upregulated upon DNA damage in a p53dependent manner. These results provide novel insight into regulation of RECQ1 expression and its role in DNA damage response. As RECQ1 efficiently protects cells from genomic instability through repair of DNA lesions including those induced by alkylating and other chemotherapeutic agents, elevated RECQ1 expression in tumor cells may provide resistance to anticancer drugs.

\section{RESULTS}

\section{Genotoxic stress upregulates $R E C Q 1$ expression}

To test whether genotoxic stress modulates RECQ1 expression, we first measured RECQ1 mRNA levels in U2OS (osteosarcoma) cells that were either untreated or treated with etoposide $(1 \mu \mathrm{M})$, doxorubicin $(500 \mathrm{nM})$ or methylmethanesulfonate (MMS, $1 \mathrm{mM}$ ) for 4,8 or $24 \mathrm{~h}$ (Figure 1A). Quantitative RT-PCR (qRT-PCR) analysis demonstrated increased RECQ1 mRNA levels (2- to 8-fold) in response to these treatments. The kinetics and magnitude of the induction varied for each genotoxic agent. For etoposide and doxorubicin, highest level of RECQ1 mRNA was observed after $24 \mathrm{~h}$ (Figure 1A). As compared to untreated cells, U2OS cells grown for $24 \mathrm{~h}$ in the presence of etoposide and doxorubicin displayed about 3 - and 8-fold increase in RECQ1 mRNA, respectively. Treatment with MMS however resulted in an early induction of RECQ1 mRNA and $\sim 5$-fold increase was observed at $4 \mathrm{~h}$ following MMS treatment (Figure 1A). In contrast to RECQ1 mRNA, these treatments did not change $\beta$-actin mRNA levels. The MMS (1 mM, $4 \mathrm{~h})$ triggered upregulation of RECQ1 mRNA (3- to 5-fold) was also observed in mouse embryonic fibroblasts (Figure 1B). Treatment with MMS (1 $\mathrm{mM}, 4 \mathrm{~h}$ ) also resulted in a significant increase $>2.5$-fold $(p<0.05)$ in RECQ1 mRNA in MCF7 cells (breast cancer) similar to U2OS cells but not in HeLa (cervical carcinoma) cells (Figure 1C).

An important factor involved in transcriptional regulation of genes after DNA damage is p53, which becomes activated by the ATM/ATR pathway upon DNA damage [32]. Analysis of RECQ1 mRNA levels in U2OS cells treated with MMS indicated a correlation with induction of the canonical p53 target p21 (CDKN1A) suggesting that p53 status may influence DNA damage triggered upregulation of RECQ1 (Figure 1C). Treatment of cells with MMS activates ATM by single strand breaks arising during the repair of alkylating DNA lesions [33]. Similarly, DNA-PK has also been implicated in MMS mediated DNA damage response [34]. To test whether DNA-damage triggered increase in RECQ1 expression requires activities of ATM and DNA-PK, we measured RECQ1 mRNA in U2OS cells in the presence of pharmacological inhibitors of ATM (ATMi, Ku55933) or DNA-PK (DNA-PKi, Nu7026) prior to MMS exposure. Incubation of U2OS cells with ATMi or DNA-PKi $(10 \mu \mathrm{M}$, $16 \mathrm{~h}$ ) alone resulted in about 2-fold increase in RECQ1 mRNA (Figure 1D) perhaps due to increased DNA damage load upon inhibiting the activities of these critical signaling kinases. Treatment with MMS failed to induce a significant increase in RECQ1 mRNA when the ATM or DNA-PK activity was inhibited (Figure 1D) indicating that both ATM and DNA-PK contribute to the observed RECQ1 upregulation following MMS induced genotoxic stress. 


\section{DNA damage induced upregulation in RECQ1 expression is p53-dependent}

The tumor suppressor p53 functions as a master transcriptional regulator of cellular responses to genotoxic stress by inducing cell cycle arrest or apoptosis [35]. To test whether $\mathrm{p} 53$ is involved in RECQ1 upregulation, we analyzed the MMS-triggered induction of RECQ1 mRNA in isogenic colon cancer cell lines proficient or deficient for p53. Treatment of p53-proficient HCT116 cells with MMS $(4 \mathrm{~h})$ resulted in a dose dependent increase in RECQ1 mRNA (Figure 2A) as measured by qRT-PCR using two independent primer sets; in contrast, no significant change in $\beta$-actin mRNA was observed in response to MMS treatment (Figure 2A). Because we observed maximum induction of RECQ1 mRNA at $1 \mathrm{mM}$ MMS, we chose this dose of MMS to compare RECQ1 mRNA levels in p53-proficient (p53 wild-type, p53WT) HCT116 cells and p53-deficient (p53 knockout, p53KO) HCT116 cells. We found that RECQ1 mRNA was induced only in p53WT-
HCT116 cells; p53KO-HCT116 cells did not upregulate RECQ1 up to after $8 \mathrm{~h}$ exposure to MMS (Figure 2B). As a positive control for p53-target, we also observed induction of p21 mRNA specifically in p53WT-HCT116 cells. In p53WT-HCT116 cells, we observed $>10$-fold increase in RECQ1 mRNA after $24 \mathrm{~h}$ of MMS treatment. Notably, p53KO-HCT116 cells displayed a mild ( 4-fold) increase in RECQ1 mRNA levels at $24 \mathrm{~h}$ (Figure 2B). This induction is likely due to p53-independent stress response since we also observed $p 21$ induction, as shown previously [36], in p53KO-HCT116 cells at $24 \mathrm{~h}$ treatment (Figure 2B). Notably, this p53-dependent upregulation of RECQ1 and $p 21 \mathrm{mRNA}$ was also observed in p53-proficient but not isogenic p53-deficient RKO cells (Figure 2C). Consistent with the increase in RECQ1 mRNA, MMS treatment also resulted in a p53-dependent increase in RECQ1 protein in HCT116 cells (Figure 2D). Furthermore, U2OS cells transfected with $\mathrm{p} 53$ siRNA failed to upregulate RECQ1 mRNA and the positive control p21 mRNA, following MMS (1 mM) treatment (Figure 2E). Collectively, these
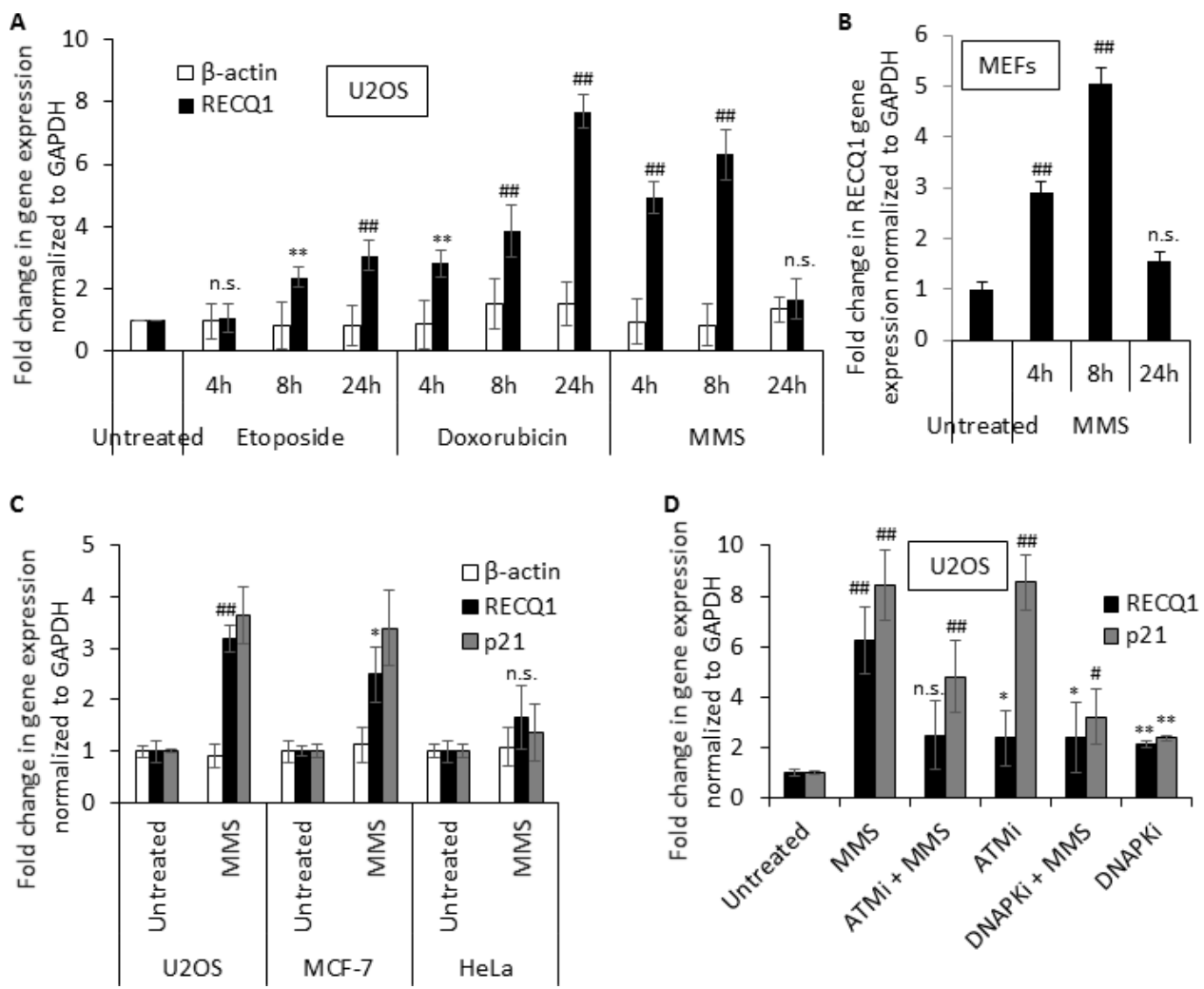

Figure 1: Genotoxic stress upregulates $\boldsymbol{R E C Q 1 ~ e x p r e s s i o n . ~ ( A ) ~ S u m m a r y ~ o f ~ q u a n t i t a t i v e - P C R ~ d a t a ~ o n ~} R E C Q 1 \mathrm{mRNA}$ in U2OS cells that were either untreated or treated with etoposide $(1 \mu \mathrm{M})$, doxorubicin $(500 \mathrm{nM})$ or MMS $(1 \mathrm{mM})$ for 4,8 or $24 \mathrm{~h}$. Change in $\beta$-actin mRNA was measured as an additional house-keeping control. (B) MMS treatment also upregulates RECQ1 in mouse embryonic fibroblasts (MEFs). (C) MMS induced upregulation of RECQ1 mRNA is not cell line specific and correlates with upregulation of $p 21$, an established p53 target. U2OS, MCF-7, or HeLa cells were untreated or treated with MMS (1 mM) for $4 \mathrm{~h}$. Fold-change in gene expression compared to untreated and normalized to GAPDH is shown. (D) MMS induced upregulation of RECQ1 mRNA in U2OS cells is dependent on activities of ATM and DNA-PK. U2OS cells were untreated or treated with pharmacological inhibitors of ATM (ATMi; $10 \mu \mathrm{M})$ or DNA-PK (DNA$\mathrm{PKi} ; 10 \mu \mathrm{M})$ for $16 \mathrm{~h}$ prior to treatment with MMS $(1 \mathrm{mM}, 4 \mathrm{~h})$. Fold-change in gene expression compared to untreated and normalized to $G A P D H$ is shown. Values are average of three independent experiments and standard deviation is indicated by error bars. Statistical significance of RECQ1 expression changes in untreated versus treatment groups is indicated as ${ }^{*} p<0.05 ;{ }^{\#} p<0.01 ;{ }^{* *} p<0.005 ;{ }^{* \#} p<$ 0.001 ; or n. s., non-significant. 
experiments demonstrate a clear p53-dependence for MMS-induced upregulation of RECQ1 mRNA.

The basal levels of RECQ1 mRNA in p53-deficient HCT116 and RKO cells were 0.6- and 0.8-fold as compared to their p53-proficient isogenic counterparts, indicating that basal RECQ1 expression level may also be p53-dependent (Supplementary Figure 1). However, basal RECQ1 protein levels were comparable in p53-WT and p53-KO HCT116 cells (Figure 4A). We also note that the MMS-induced upregulation of RECQ1 protein did not follow the same kinetics as the mRNA in p53-proficient HCT116 (Figure 2D). At this point, it is difficult to correlate the regulation at the mRNA level to the changes in protein levels. It is possible that RECQ1 is regulated both at the transcriptional and posttranscriptional levels. We also analyzed mRNA levels of all five human RecQ homologs in HCT116 and RKO cells for DNA damage and p53 dependence (Supplementary Figure 2). Consistent with
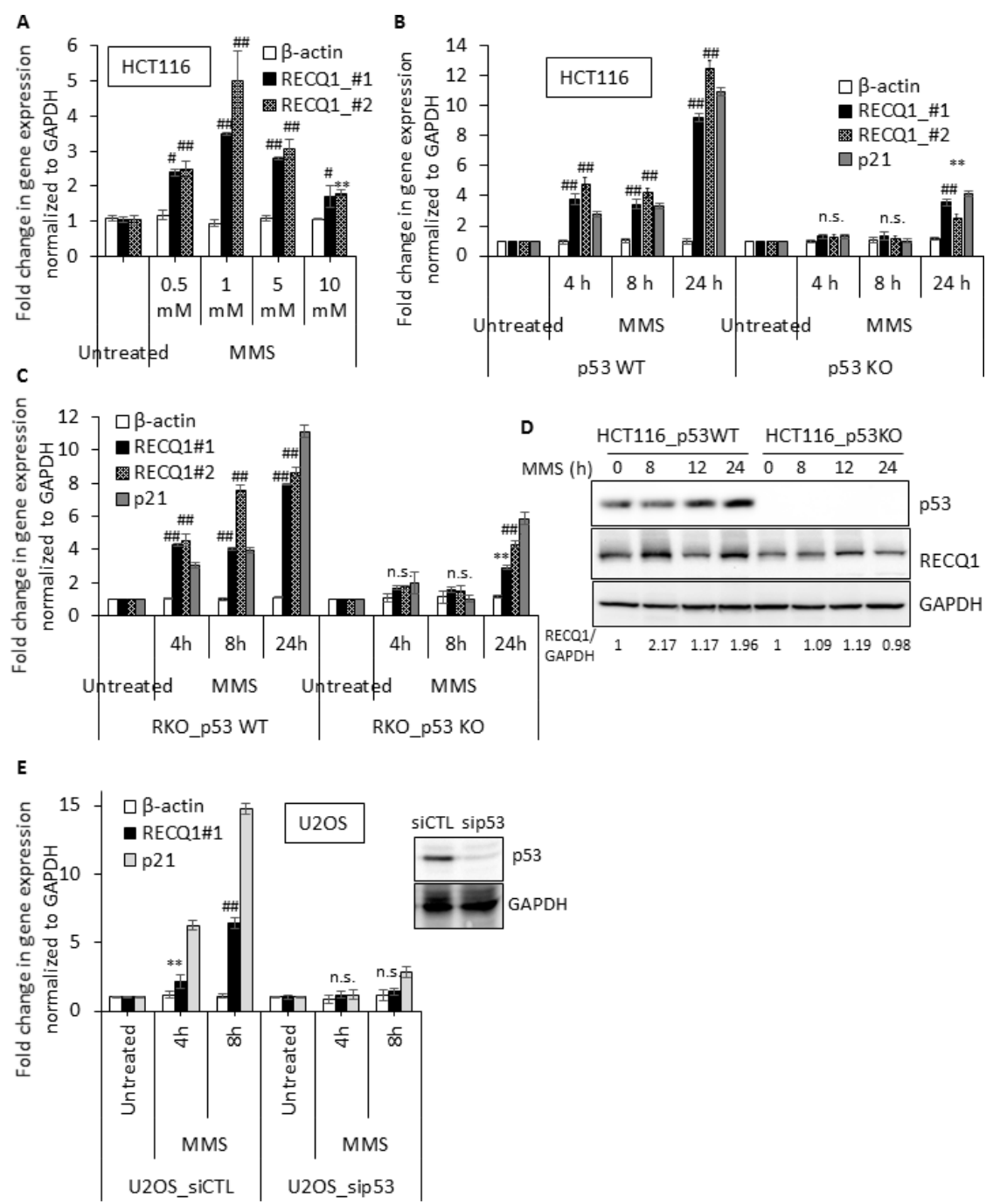

Figure 2: MMS induced upregulation of RECQ1 is p53 dependent. (A) $R E C Q 1$ expression is upregulated in response to MMS treatment in HCT116 cells expressing wild-type p53. Cells were either untreated or treated for $4 \mathrm{~h}$ with the indicated dose of MMS. Foldchange in gene expression compared to untreated and normalized to GAPDH is shown. Two primer sets (RECQ1 \#1, and RECQ1 \#2) were used for measuring RECQ1 mRNA. $\beta$-actin served as an additional housekeeping control. (B) Isogenic HCT116 cells expressing either the wild-type p53 (p53 WT) or knockout for p53 (p53 KO) were exposed to MMS (1 mM) for indicated time period and the fold-change in mRNA expression of RECQ1 and $p 21$ compared to untreated as measured by qPCR is shown. (C) Fold-change in mRNA expression of RECQ1 and $p 21$ compared to untreated in isogenic p53WT and p53KO RKO cells is shown. (D) Following treatment with MMS (1 mM) for indicated time, expression of RECQ1 and p53 proteins was determined by Western blot analysis of whole-cell extracts. GAPDH was used as a loading control and fold-change in RECQ1 protein expression normalized to GAPDH is indicated. (E) U2OS cells, $42 \mathrm{~h}$ after transfection with control siRNA or p53 siRNA, were exposed to MMS (1 mM) for indicated time period and the fold-change in mRNA expression of RECQ1 and $p 21$ compared to untreated cells as measured by qPCR is shown. $\beta$-actin served as an additional housekeeping control. Knockdown of p53 protein level is shown by Western Blot. For all the qPCR data, values are average of three independent experiments and standard deviation is indicated by error bars. Statistical significance of $R E C Q 1$ expression changes in untreated versus treatment groups is indicated as ${ }^{*} p<0.05 ;{ }^{*} p<0.01 ; * * p<0.005$; ${ }^{*} p<0.001$; or n. s., non-significant. 
a previous report of transcriptional repression of RECQ4 by p53 [37], MMS-treated p53-deficient cells displayed elevated RECQ4 mRNA (Supplementary Figure 2). We did not observe significant changes in expression of $B L M$ or RECQ5 in response to MMS treatment, however $W R N$ expression was upregulated in p53-dependent fashion (Supplementary Figure 2). In p53WT-HCT116 cells, about 6-fold and 2-fold higher $W R N$ mRNA was measured at 4 and $24 \mathrm{~h}$ MMS treatment $(1 \mathrm{mM})$ as compared to untreated cells. In contrast, about 4-fold and 10-fold increase in RECQ1 mRNA was measured at 4 and $24 \mathrm{~h}$ MMS treatment (1 mM), respectively (Supplementary Figure 2). Distinct kinetics and p53-dependence of RECQ1, WRN and RECQ4 mRNA upregulation suggests that encoded proteins participate at different steps and/or pathways for cellular response to MMS treatment.

\section{The RECQ1 promoter-luciferase reporter is responsive to $\mathrm{p53}$}

Having identified that MMS-induced RECQ1 upregulation is p53-dependent, we sought to determine if RECQ1 is a direct target of $\mathrm{p} 53$. Transcription regulation by $\mathrm{p} 53$ is enacted through its binding to the consensus sequence motif RRRCWWGYYY $\left(\mathrm{N}_{0-13}\right)$ RRRCWWGYYY where $\mathrm{R}$ is a purine, $\mathrm{W}$ is an adenine or thymine, $\mathrm{Y}$ is a pyrimidine and $\mathrm{N}$ is any base [38]. Additionally, p53 can bind to non-canonical response elements (REs) or half and three-quarter consensus sequences to mediate transactivation of several genes including those involved in DNA repair responses [39]. Analysis using the IARC Tp53 database (http://p53.iarc.fr/TargetGenes. aspx?\&sq=p53+response+element) suggested p53binding sites in the RECQ1 promoter. We searched for the potential binding sites of $\mathrm{p} 53$ in a region $\sim 1 \mathrm{~kb}$ upstream and downstream of the human RECQ1 transcriptional start site using Promo 3.0.2 online tool (http://alggen.lsi.upc.es/ cgi-bin/promo_v3/promo/promoinit.cgi?dirDB=TF_8.3)

[40] and identified multiple putative p53-binding sites, majority of which are located within 819 bp upstream of transcription start site and $21 \mathrm{bp}$ of exon 1 (Figure 3A). Of note, and as previously reported for $\mathrm{p} 53$, these predicted sites deviate from the consensus p53RE. To test the hypothesis that $\mathrm{p} 53$ promotes $R E C Q 1$ transcription, we first utilized a luciferase-reporter system employing the pGL3-Basic promoterless vector or pGL3-carrying a 625 bp RECQ1 promoter fragment containing the putative $\mathrm{p} 53$ binding sites and analyzed luciferase expression in the p53proficient and p53-deficient HCT116 cells. Our reporter assay also included a pGL3-p53RE that contained 11 consensus p53 response elements as positive control [41]. We observed that at both 24 and $48 \mathrm{~h}$ after transfection, luciferase expression from the RECQ1 promoter-luciferase reporter was significantly higher in p53WT- HCT116 cells as compared to p53KO- HCT116 cells (Figure 3B). As a positive control, luciferase expression of pGL3-p53RE was also significantly reduced in p53KO-HCT116 cells whereas the luciferase activity of pGL3-empty vector remained comparable in p53-deficient or p53-proficient cells (Figure 3B). This data indicates that basal RECQ1 promoter activity is driven by $\mathrm{p} 53$ and is consistent with our data showing that endogenous basal RECQ1 mRNA levels are higher in p53WT-HCT116 cells as compared to p53KO-HCT116 cells (Supplementary Figure 1).

\section{DNA damage recruits p53 to the RECQ1 promoter}

We next employed chromatin immunoprecipitation (ChIP) experiments in conjunction with qPCR to determine if endogenous p53 associates with the predicted binding sites in the RECQ1 promoter. Crosslinked chromatin from p53-proficient or p53-deficient HCT116 cells, untreated or treated with MMS ( $1 \mathrm{mM}, 8$ h) was immunoprecipitated with a control IgG or specific antibody against p53 (DO-1). Following cross-link reversal, the immunoprecipitated chromatin was subjected to qPCR to determine the enrichment of RECQ1-promoter sequence using three independent primer sets spanning the predicted p53 binding sites within RECQ1 promoter. In untreated p53WT- HCT116 cells, we found $\sim 2$-fold enrichment of $\mathrm{p} 53$ at the $R E C Q 1$ promoter containing putative p53 binding sequences (Figure 3C). Treatment with MMS resulted in a marked increase in p53 binding at the RECQ1 promoter; we observed $~ 3-, 7-$, and 11-fold enrichment in $\mathrm{p} 53$ binding at RECQ1 promoter site 1,2 and 3, respectively in p53WT-HCT116 cells (Figure 3C). As a positive control for the p53-ChIP assay, we confirmed p53 binding to a known p53RE of $p 21$ [41], a downstream target of $\mathrm{p} 53$, in p53WT-HCT116 cells (Figure 3C). MMS treatment also resulted in 6-fold enrichment of p53 at the $p 21$ promoter as compared to 2 -fold enrichment in untreated p53WT- HCT116 cells (Figure 3C). In contrast, the RECQ1 promoter or the $p 21$ promoter was not enriched in the $\mathrm{p} 53-\mathrm{ChIP}$ material in $\mathrm{p} 53 \mathrm{KO}-\mathrm{HCT} 116$ cells indicating that the observed enrichment of the RECQ1 and p21 promoter in the p53-ChIP material in HCT116-p53WT cells was specific to p53 (Figure 3C, 3D). In untreated or MMS-treated condition, we did not observe enrichment of a negative control genomic region corresponding to GAPDH in the p53-ChIP over IgG-ChIP, (Figure 3C, 3D) demonstrating specificity of the interaction between $\mathrm{p} 53$ and the p53-response elements in the RECQ1 promoter. Chromatin immunoprecipitation with RNA polymerase (Pol) II antibody demonstrated a p53-dependent and significantly enhanced recruitment of RNA Pol II to the promoter regions of RECQ1 and $p 21$ upon MMS treatment suggesting transcriptional upregulation (Figure 3E). Binding of $\mathrm{p} 53$ to the RECQ1 promoter and the enhanced binding following MMS treatment was also observed in U2OS cells (Supplementary Figure $3 \mathrm{~A})$. We also observed binding in these regions of the 
A

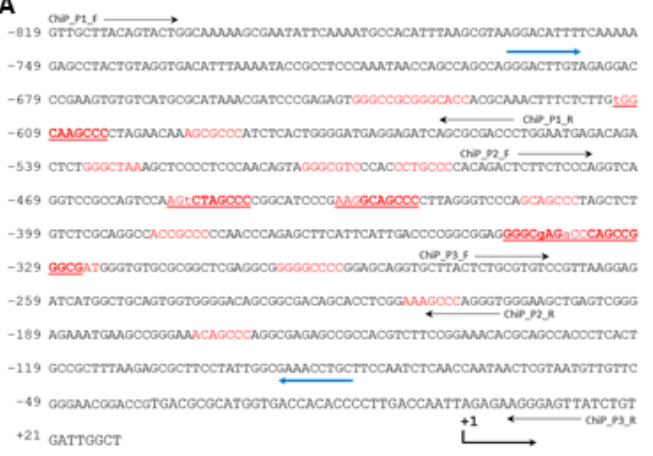

c

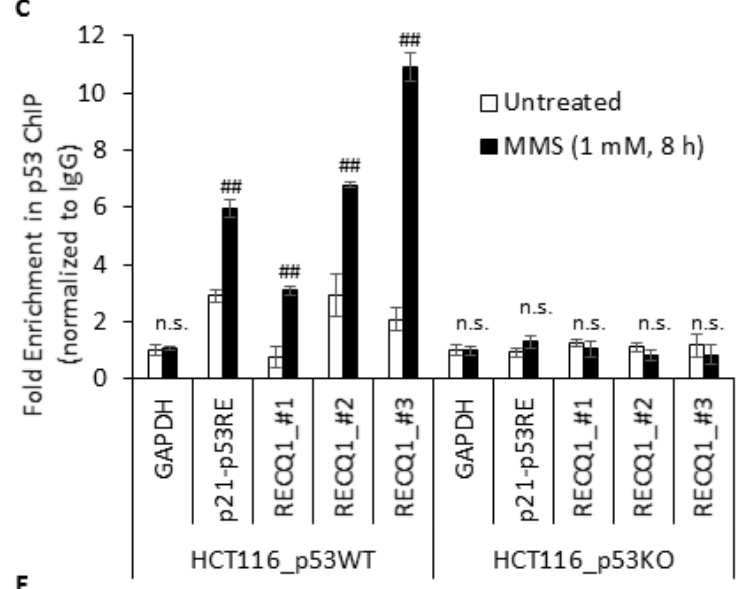

$\mathbf{E}$

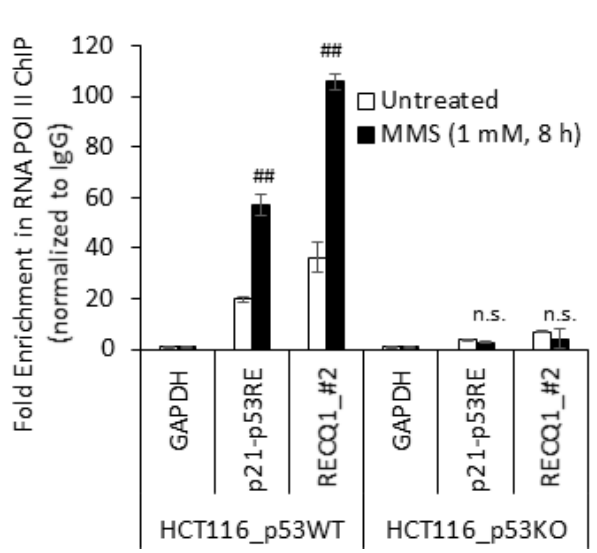

B

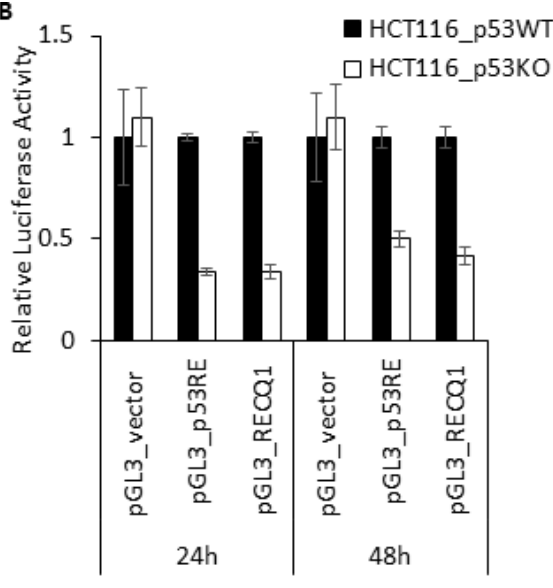

D

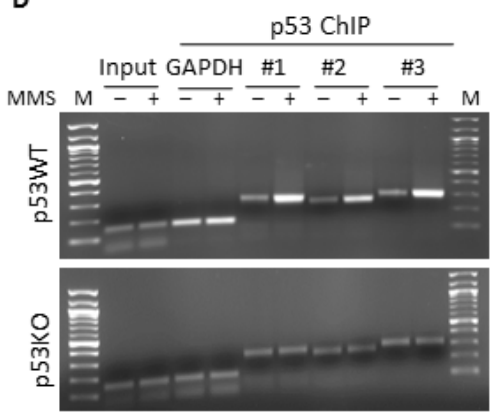

Figure 3: 553 is enriched at the $R E C Q 1$ promoter following MMS treatment. (A) Partial sequence of the $R E C Q 1$ promoter $(-819 \mathrm{bp}$ to $+21 \mathrm{bp})$ is shown with potential p53-binding sites predicted by Promo 3.0.2 indicated in red, those predicted by Tp53 database are underlined, and those predicted by both Promo 3.0.2 and Tp53 are indicated in bold. Transcriptional start site is indicated as +1 . The position of primer used for PCR-cloning of a $625 \mathrm{bp}$ fragment from RECQ1 promoter in pGL3-Basic for luciferase assay is indicated by blue arrow. Three primer sets used for ChIP-qPCR analyses of p53 binding are indicated. (B) Dual Luciferase Assay shows p53-dependent transcriptional activation of a $625 \mathrm{bp}$ fragment from RECQ1 promoter region; pGL3 p53RE served as a positive control and pGL3_vector as a negative control. The relative luciferase activity was first determined by ratio of firefly and renilla luciferase activity for each sample in p53-WT and p53-KO HCT116 cells, and the relative promoter activity in p53-KO was calculated using the relative luciferase activity from p53-WT cells transfected by each pGL3-basic construct as a reference of 1 . Bars indicate mean values plus standard deviation of three independent experiments. (C) MMS-induced enrichment of $\mathrm{p} 53$ to RECQ1 promoter. HCT116 cells, p53-WT or p53-KO, untreated or treated with MMS ( $1 \mathrm{mM}, 8 \mathrm{~h})$, were processed for ChIP using a p53-specific antibody. ChIP experiments with rabbit IgG served as negative control. ChIP-qPCR of immunoprecipitated DNA with three probes specific for $R E C Q 1$ promoter sequence containing predicted p53 binding sites (\#1, \#2, and \#3, and as shown in A). Binding of p53 to $p 21$ promoter containing p53RE served as a positive control. Fold enrichment over IgG was determined and is shown for each primer pair for the ChIP. Relative occupancy at RECQ1 and $p 21$ promoter sequence versus a negative control site DNA containing GAPDH shows MMS treatment induced enrichment of p53. Statistical significance of enrichment in untreated versus treatment groups is indicated. Results are expressed as means \pm SEM for at least three independent experiments. (D) A 
representative agarose gel of the amplified DNA immunoprecipitated with p53 antibody shows MMS-induced enrichment of p53 to RECQ1 promoter whereas MMS treatment did not change GAPDH abundance in p53 ChIP. M, DNA size marker. (E) Enhanced recruitment of RNA POL II to RECQ1 promoter following MMS treatment. HCT116 cells, p53-WT or p53-KO, untreated or treated with MMS (1 mM, $8 \mathrm{~h}$ ), were processed for ChIP using a RNA POL II-specific antibody or rabbit IgG. Binding of RNA POL II to RECQ1 promoter sequence and $p 21$ promoter was measured by qPCR of immunoprecipitated DNA. Fold enrichment over IgG was determined and is shown for each primer pair for the ChIP. Relative occupancy at RECQ1 and $p 21$ promoter sequence versus a negative control non-promoter GAPDH sequence shows MMS treatment induced enrichment of RNA POL II. Statistical significance of enrichment in untreated versus treatment groups is indicated. Results are expressed as means \pm SEM for at least three independent experiments.

RECQ1 promoter corresponding to the p53 ChIP-seq peak in data from U2OS cells published by others [42] (Supplementary Figure 3B-3D). Our results suggest in vivo association of $\mathrm{p} 53$ with RECQ1 promoter is enriched upon MMS-induced DNA damage. Overall, the presence of $\mathrm{p} 53$ binding site, and the observed MMS-induced enrichment of p53 at the RECQ1 promoter is consistent with the hypothesis that $\mathrm{p} 53$ mediates the upregulation of $R E C Q 1$ after DNA damage.

\section{RECQ1 promotes DNA repair and survival following MMS-treatment}

Given the well-established roles of p53 in genotoxic stress response, we sought to determine the effect of RECQ1-depletion in p53-proficient and p53-deficient HCT116 cells on survival after MMS treatment. We transfected these cells with a control (CTL) siRNA or RECQ1 siRNAs $(20 \mathrm{nM})$ for $48 \mathrm{~h}$ and validated significant knockdown of RECQ1 protein (>90\%) by immunoblotting (Figure 4A). Following $48 \mathrm{~h}$ of transfection, CTL or RECQ1 siRNA-transfected cells were exposed to increasing concentrations of MMS for 24 $\mathrm{h}$ and cell survival was measured by trypan blue exclusion assay following a $24 \mathrm{~h}$ recovery in drug free medium (Figure 4B). As reported previously [43], p53KO-HCT116 cells were more sensitive to MMS treatment than p53WTHCT116 cells. As compared to CTL siRNA transfected cells, RECQ1-depletion in p53WT-HCT116 cells also resulted in increased MMS sensitivity; however, RECQ1depletion in p53KO-HCT116 cells caused maximum sensitivity to MMS (Figure 4B). This synergistic effect on survival upon concurrent deficiency of RECQ1 and p53 indicates their parallel roles in DNA damage response to MMS. It is also likely that compensatory mechanisms operate in the combined absence of RECQ1 and p53 since the depletion of RECQ1 in p53-KO cells did not show a dramatic reduction in survival.

Similar to our observation in HCT116 cells, U2OS cells stably transduced with a RECQ1 shRNA were more sensitive than those transduced with a CTL (luciferase)shRNA over a range of MMS concentration tested (Figure 4C). This data demonstrates that the increased sensitivity to MMS is not cell-type specific and is sustained in cells depleted of RECQ1 over a longer period of time. MMS is known to produce heat-labile DNA damage repaired by base excision repair, but no detectable in vivo DNA double strand breaks [44]. DNA alkylation due to MMS also blocks replication fork elongation in mammalian cells, causing formation of replicationassociated DNA lesions [45]. The cellular level of RECQ1 protein modulates overall DNA damage [22] and RECQ1 functions in replication restart following stress [25]. Thus, we next examined $\gamma \mathrm{H} 2 \mathrm{AX}$ as a surrogate marker of DNA double strand breaks in CTL and RECQ1-knockdown U2OS cells exposed to MMS ( $1 \mathrm{mM}$, 4h) or Doxorubicin $(1 \mu \mathrm{M}, 4 \mathrm{~h})$, a topoisomerase II-stabilizing drug known to cause double strand breaks in DNA [46]. At the protein level, increased RECQ1 levels were observed in control U2OS cells upon treatment with MMS and doxorubicin (Figure 4D); and this increase was more readily noticeable in short exposure due to constitutively high abundance of RECQ1 protein in total cell lysate used in Western Blots. The increased RECQ1 protein is consistent with the observed upregulation of RECQ1 mRNA in U2OS cells (Figure 1A). In control U2OS cells, MMS treatment did not result in significant double strand breaks as detected by $\gamma \mathrm{H} 2 \mathrm{AX}$ signal. In contrast, RECQ1-knockdown U2OS cells demonstrated greater $\gamma \mathrm{H} 2 \mathrm{AX}$ signal after treatment with MMS (Figure 4D). As compared to control U2OS cells, RECQ1-knockdown cells also exhibited significantly greater $\gamma \mathrm{H} 2 \mathrm{AX}$ after doxorubicin treatment (Figure 4D). As reported previously [46], doxorubicin treatment stabilized p53 and increased p21 levels indicating transcriptional activation; RECQ1-knockdown cells displayed greater signal for p53 and p21 proteins as compared to control U2OS cells (Figure 4D). Since $\gamma \mathrm{H} 2 \mathrm{AX}$ is formed in response to direct or indirectly-produced double strand breaks, the presence of double strand breaks was also measured using a neutral comet assay. We determined tail moment, a measure of the amount and distance of DNA migration, in control and RECQ1-knockdown U2OS cells. As compared to untreated condition, treatment with Doxorubicin $(1 \mu \mathrm{M}$, $4 \mathrm{~h})$ or MMS (1 mM, $4 \mathrm{~h})$ resulted in increased tail moment in U2OS cells (Figure 4E). Consistent with the observed increase in $\gamma \mathrm{H} 2 \mathrm{AX}$, RECQ1-knockdown U2OS cells exhibited increased mean tail moment as compared to control U2OS cells, indicating higher levels of double strand breaks when exposed to doxorubicin or MMS in neutral comet assays (Figure 4E).

Overall, increased accumulation and transactivation of p53, and increased double strand breaks are consistent with the observed sensitivity of RECQ1-deficienct cells to MMS as shown here and doxorubicin as recently reported [47]. 


\section{RECQ1 expression positively correlates with cellular resistance to clinically relevant DNA damaging agents}

RECQ1 deficiency leads to genomic instability and sensitivity to a range of genotoxins $[4,5]$. Given the sensitivity of RECQ1-deficient cells to MMS, we first examined whether $R E C Q 1$ expression is upregulated at the mRNA level after treatment with clinically relevant alkylating agents Temozolomide (TMZ) and Fotemustine (FMS), both of which are used as cancer chemotherapeutics [48]. Following $8 \mathrm{~h}$ treatment of p53WT-HCT116 cells with TMZ, > 4-fold and 6-fold increase in RECQ1 mRNA was observed in cells treated with $0.5 \mathrm{mM}$ and $1 \mathrm{mM}$
TMZ, respectively (Figure 5A). Treatment with FMS also upregulated RECQ1 mRNA in HCT116 cells, and a comparable increase (> 4-fold) in RECQ1 mRNA was observed after $8 \mathrm{~h}$ treatment with $32 \mu \mathrm{M}$ or $64 \mu \mathrm{M}$ FMS (Figure 5B). As observed for the MMS sensitivity, p53KOHCT116 cells were more sensitive for TMZ and RECQ1depletion led to increased sensitivity in both $\mathrm{p} 53$-proficient and p53-deficient HCT116 cells (Figure 5C). Moreover, treatment with TMZ $(1 \mathrm{mM}, 8 \mathrm{~h})$ or FMS $(32 \mu \mathrm{M}, 8 \mathrm{~h})$ also upregulated RECQ1 mRNA in U2OS cells (Figure 5D); and RECQ1-knockdown cells were more sensitive than control U2OS cells over a range of TMZ and FMS concentration tested (Figure 5E, 5F). Presence of TMZ (100 $\mu$ M, 5 days) in growth medium resulted in increased RECQ1 protein level
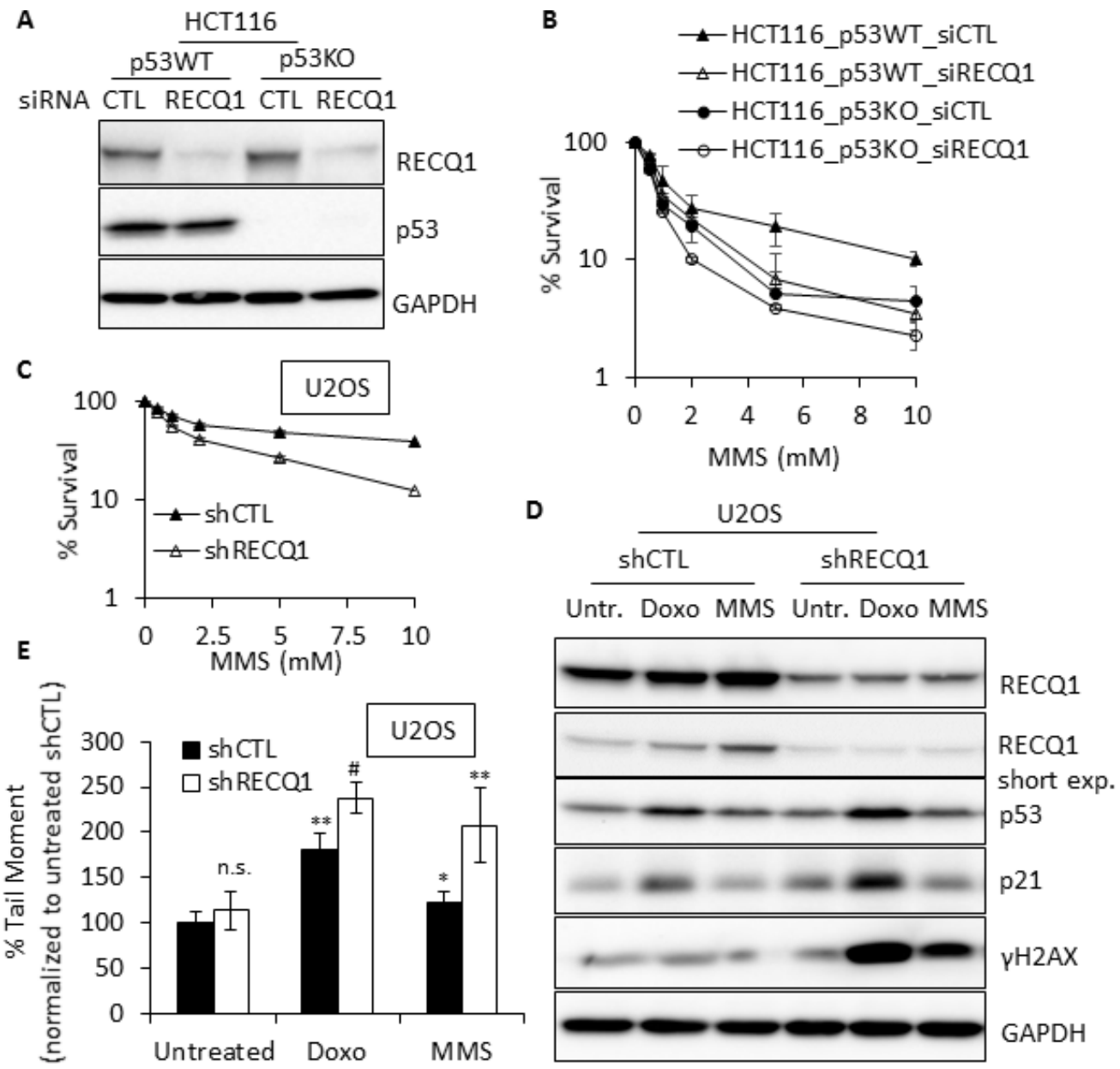

Figure 4: RECQ1 promotes DNA repair and survival after MMS treatment. (A) Western blot showing siRNA knockdown of RECQ1 in p53-proficient and p53-deficient HCT116 cells. (B) RECQ1-depletion and p53 loss have synergistic effect on survival. Following $48 \mathrm{~h}$ of siRNA transfection, p53WT and p53KO-HCT116 cells were exposed to MMS for $24 \mathrm{~h}$ and subsequently grown for 24 $\mathrm{h}$ in drug-free medium. Surviving fraction compared to untreated was determined by cell count. Knockdown of RECQ1 was confirmed by Western blotting as shown. (C) U2OS cells stably transduced with a control (shCTL) or RECQ1 (shRECQ1)-specific shRNA were exposed to MMS for $24 \mathrm{~h}$ and subsequently grown for $24 \mathrm{~h}$ in drug-free medium. Surviving fraction compared to untreated was determined by cell count. (D) Western blot analysis of whole cell extracts of stable control and RECQ1 knockdown U2OS cells, untreated or treated with doxorubicin $(1 \mu \mathrm{M})$ or MMS $(1 \mathrm{mM})$ for $4 \mathrm{~h}$. A short exposure of RECQ1 Western blot is also included. (E) DNA double strand breaks in control or RECQ1-depleted cells. Neutral Comet Assay was used to determine tail moment as a measure of double strand breaks in stable control and RECQ1 knockdown U2OS cells, untreated or treated with doxorubicin $(1 \mu \mathrm{M})$ or MMS $(1 \mathrm{mM})$ for $4 \mathrm{~h}$. Mean tail moment of untreated shCTL was used to normalize the data and is shown as $100 \%$. Statistical significance of difference in tail moment as compared to untreated shCTL is indicated as $* p<0.05 ;{ }^{*} p<0.01 ; * * p<0.005$ or n. s., non-significant. 
in control U2OS and MCF7 cells, and led to cleaved PARP in RECQ1 knockdown U2OS and MCF7 cells indicating enhanced apoptosis (Figure 5G). These results collectively demonstrate that DNA alkylating agents including the laboratory agent MMS and chemotherapeutics TMZ or FMS induce upregulation of RECQ1 mRNA, and RECQ1 expression level correlates with cellular sensitivity to these treatments. As compared to control untreated U2OS cells,
RECQ1 mRNA was also upregulated following treatment with chemotherapeutics 5-fluoro uracil (5-FU), gemcitabine, camptothecin as well as the laboratory carcinogen methylnitronitrosoguanidine (MNNG) and environmental mutagen benzo[a]pyrene (Supplementary Figure 4). This is consistent with previous studies where RECQ1-depletion increased cellular sensitivity to camptothecin and benzo[a] pyrene $[22,49]$.
A
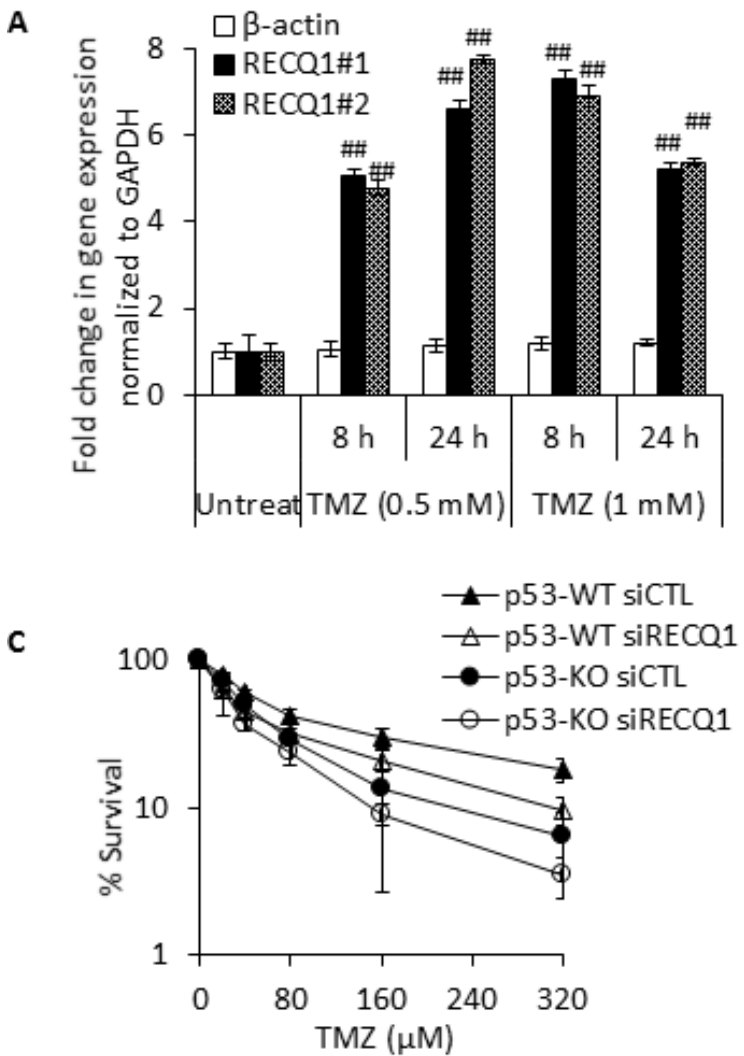

E

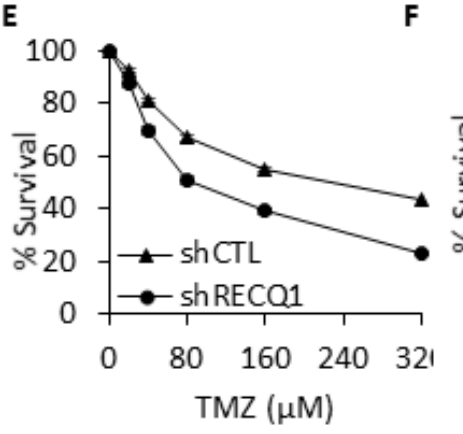

F

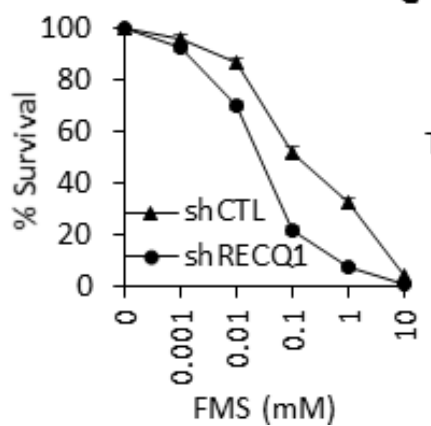

G
B

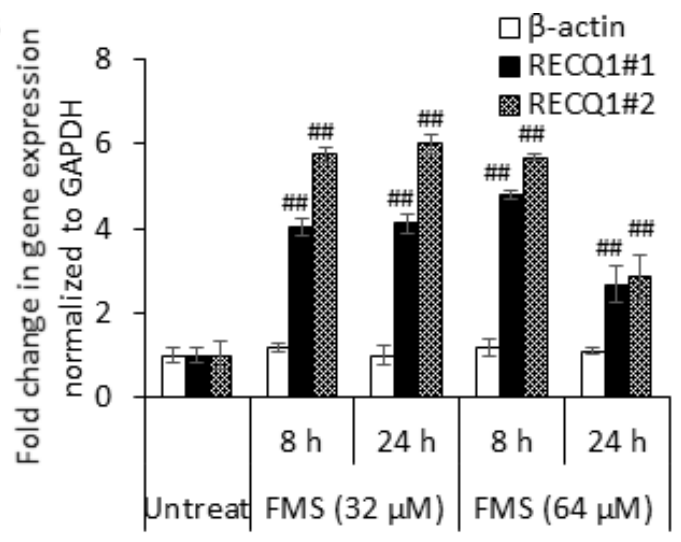

D
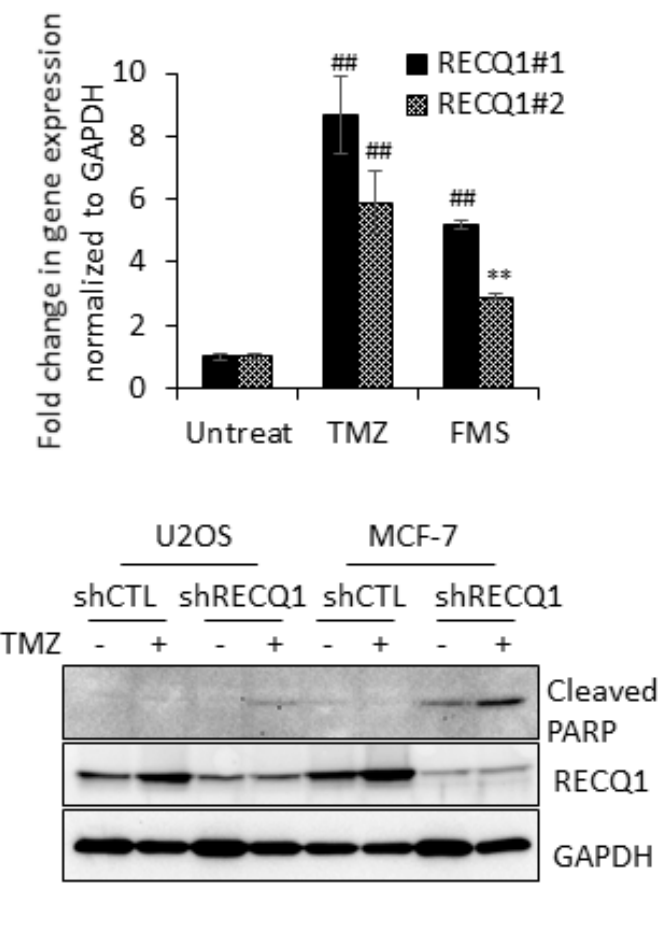

Figure 5: RECQ1 expression is upregulated by the chemotherapeutic drugs that alkylate DNA and positively correlates with cellular resistance to these treatments. (A, B) RECQ1 expression is upregulated in response to Temozolomide (TMZ) or Fotemustine (FMS) treatment of HCT116 cells expressing wild-type p53. RECQ1 mRNA expression was measured using two independent primer sets; fold-change in expression compared to untreated and normalized to GAPDH is shown. $\beta$-actin served as an additional housekeeping control. Values are average of three independent experiments and standard deviation is indicated by error bars. Statistical significance of RECQ1 expression changes in untreated versus treatment groups is indicated as ${ }^{*} p<0.05 ;{ }^{*} p<0.01 ; * * p<0.005$; ${ }^{\#} p<0.001$; or n. s., non-significant. (C) Following 48 h of control or RECQ1 siRNA transfection, p53WT and p53KO-HCT116 cells were exposed to increasing dose of TMZ for $24 \mathrm{~h}$ and subsequently grown for $24 \mathrm{~h}$ in drug-free medium. Surviving fraction compared to untreated was determined by cell count. Knockdown of RECQ1 was confirmed by Western blotting (not shown). Surviving fraction values are the mean $\pm \mathrm{SEM}$ from three independent experiments. (D) RECQ1 expression is also upregulated in U2OS cells following treatment with TMZ ( $1 \mathrm{mM}, 6 \mathrm{~h})$ or FMS $(32 \mu \mathrm{M}, 6 \mathrm{~h})$. Fold-change in expression compared to untreated and normalized to GAPDH is shown. Values 
are average of three independent experiments and standard deviation is indicated by error bars. Statistical significance of change in $R E C Q 1$ expression compared to untreated is indicated. (E, F) Stable control (shCTL) and RECQ1 knockdown (shRECQ1) U2OS cells were exposed to increasing dose of TMZ or FMS for $24 \mathrm{~h}$ and subsequently grown for a further $24 \mathrm{~h}$ in drug-free medium. The graphs show the cellular surviving fractions measured at different doses of drug treatment in control and RECQ1-depleted cells. Surviving fraction values are the mean \pm SEM from three independent experiments. (G) Whole cell extracts prepared from stable control and RECQ1 knockdown U2OS and MCF7 cells cultured for 5 days in the absence or presence of TMZ (100 $\mu \mathrm{M})$ were subjected to Western blot analysis of cleaved PARP for apoptosis. Knockdown of RECQ1 was verified by Western blotting. TMZ treatment lead to increase in RECQ1 protein level in both U2OS and MCF7 cells transduced with control shRNA.

\section{Genotoxic stress-induced upregulation of RECQ1 mRNA expression in MDA-MB-231 cells}

p53 is a well-known tumor suppressor and p53 mutations are by far the most common in human cancer, including breast cancer. MDA-MB-231 human breast cancer cells express mutant p53 (p53R280K) that confers a "gain of function" including chemotherapy resistance, metabolic deregulation, and increased metastasis. Unlike wild-type p53 that primarily mediates its effects through sequence-specific DNA binding to cognate 553 response elements located in the promoters of p53 target genes, the mutant $\mathrm{p} 53$ protein generally has diminished wild-type $\mathrm{p} 53$ activity but it regulates gene expression via interactions with other transcription factors [50-53]. Interestingly, we found that DNA damage also upregulated RECQ1 mRNA in MDA-MB-231 cells (Figure 6A). To determine whether mutant p53 affected the expression of RECQ1, we utilized the CRISPR/Cas9 technology to knockout mutant p53 in MDA-MB-231 cells (Supplementary Figure 5). After confirming successful targeted deletion by Sanger sequencing (data not shown), we performed immunoblotting. As shown in Figure 6B, mutant p53 protein was lost in the knockout clone (p53_KO\#1) but it was robustly expressed in an isogenic clone in which the mutant p53 gene was intact (mutp53). MMS treatment resulted in a 5-fold increase in RECQ1 mRNA in the mutp53-expressing clone whereas no significant change in RECQ1 mRNA was observed in p53_KO\#1 clone (Figure 6C). Furthermore, p53-ChIP in MDA-MB-231 cells recovered $R E C Q 1$ promoter regions indicating that mutant p53 associates with the RECQ1 promoter (Figure 6D). These data indicate that RECQ1 mRNA is induced by DNA damage in a mutant p53-dependent manner in MDAMB-231 cells.

\section{Clinical correlation of RECQ1 expression in human cancer}

Germline mutations in RECQ1 significantly enhance lifetime risk of developing breast cancer $[9,10]$. In the Cancer Genome Atlas (TCGA), out of 817 patients analyzed for the Breast Invasive Carcinoma [54], 68 samples showed RECQ1 mRNA upregulation whereas only 7 showed downregulated RECQ1 mRNA. In addition, the $R E C Q 1$ gene is affected also by copy number alterations, with 3 patients showing a deep deletion and 17 patients showing an amplification of which 14 also showed RECQ1 overexpression (Supplementary Figure 6A). In this dataset, carriers of altered RECQ1 copy number and mRNA expression show a significantly shorter overall survival (Supplementary Figure 6B). Analysis of TCGA data shows significant association of $R E C Q 1$ alterations with p53 mutation $(p<0.001)$. Similarly, RECQ1 alterations exhibit significant tendency to co-occur with p53 mutations in METABRIC cohort of breast cancer patients where RECQ1 expression is correlated with patient survival [47]. To investigate whether RECQ1 mRNA expression predicts response to chemotherapy, we explored a large gene expression data set of 3951 human breast tumors (http:// kmplot.com/analysis/index.php? $\mathrm{p}=$ service $\&$ default=true) [55]. In the whole cohort that received chemotherapy, high RECQ1 mRNA was associated with poor survival (Figure 7B) $(p=0.00005)$. In patients who received no chemotherapy, high RECQ1 mRNA was also associated with poor survival (Figure 7A), but this association was less significant $(p=0.022)$. In p53 wild-type tumors, high RECQ1 mRNA was associated with poor survival in patients who received no chemotherapy (Figure 7C) $(p=0.027)$ and was borderline non-significant in patients who received chemotherapy (Figure 7D) $(p=0.085)$. These data may be consistent with our result from cell lines where we found that in $\mathrm{p} 53$ wild-type cells, knockdown of RECQ1 results in increased sensitivity to DNA damage. Interestingly in p53 mutant tumors, we observed the opposite. Low RECQ1 mRNA was significantly associated with poor survival in patients who received chemotherapy (Figure 7F) $(p=0.004)$ and was borderline non-significant in patients who received no chemotherapy (Figure 7E) $(p=0.072)$. Taken together, these clinical data suggest that the prognostic and predictive significance of RECQ1 expression may be influenced by p53 status in breast cancers.

\section{DISCUSSION}

Our results demonstrate upregulation of endogenous RECQ1 upon exposure to DNA damaging agents. We found that the DNA damage induced upregulation of RECQ1 mRNA is $\mathrm{p} 53$-dependent which binds and activates the RECQ1 promoter. We demonstrate that induction of RECQ1 by DNA damage occurs in p53-proficient HCT116 cells at the level of mRNA and protein. RECQ1 expression is also upregulated in cells exposed to TMZ, FMS, and doxorubicin; and correlates positively with cell survival following treatment with these agents. Induction of 
RECQ1 mRNA by genotoxins was seen in osteosarcoma, breast cancer, and colon cancer cell lines. Our observation that mouse embryonic fibroblasts also upregulated $R E C Q 1$ expression in response to DNA damage is consistent with its DNA repair function and suggests that RECQ1 may protect normal cells from the DNA damaging effects of anticancer drugs. Upregulation of RECQ1 expression in cancer cells, however, may contribute to chemoresistance.
Our findings are consistent with a previous proteome-wide study where etoposide treatment increased the abundance of RECQ1 peptides specifically in p53-proficient cells but not in p53-deficient cells [56]. This is the first report of RECQ1 as being p53-regulated transcriptional target, although a distinct role for RECQ1 has been described in the maintenance of the genomic stability [57]. RECQ1 catalyzes DNA unwinding and strand annealing [58], and
A

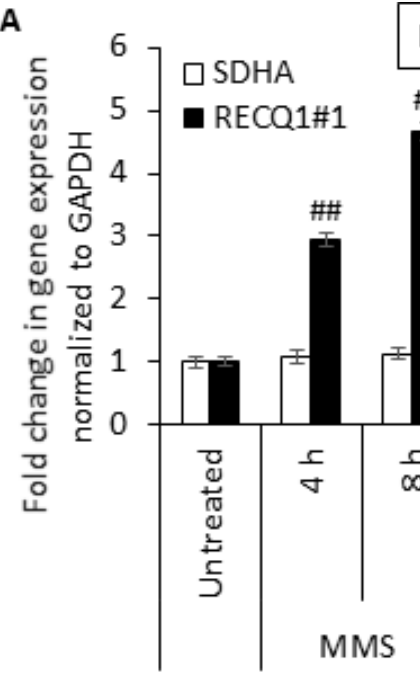

C

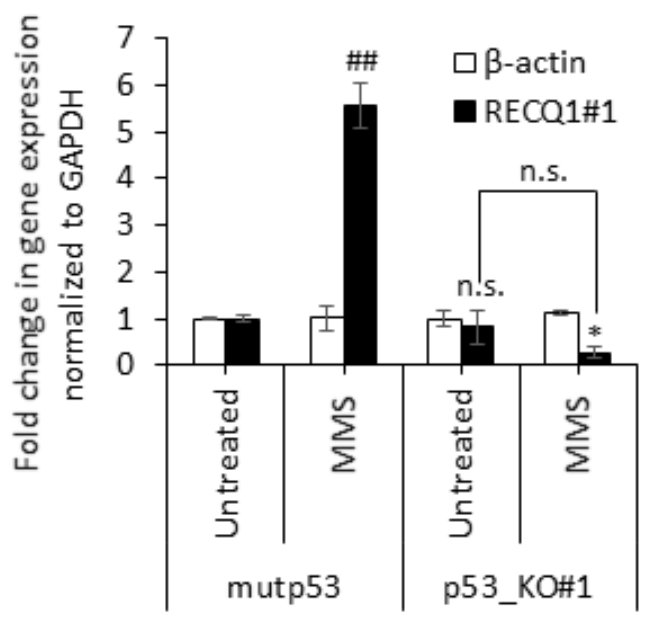

MDA-MB-231

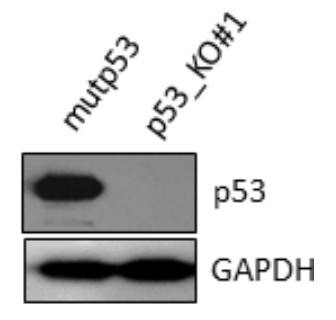

MDA-MB-231

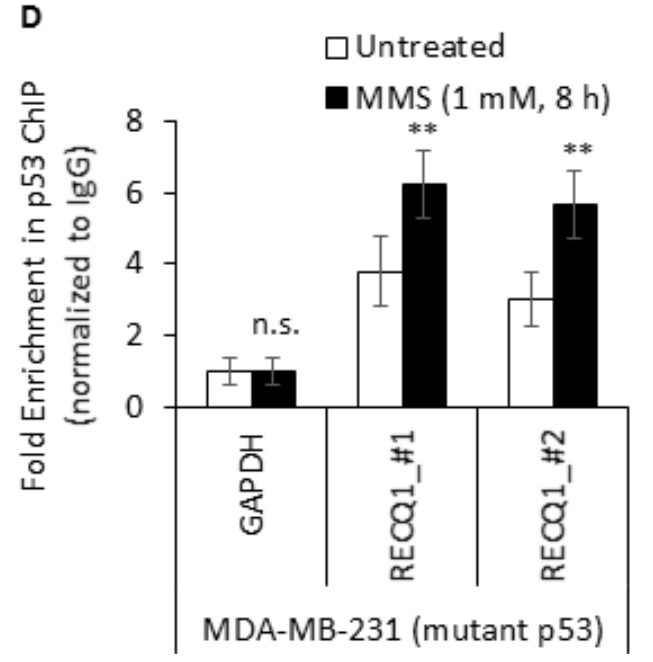

Figure 6: DNA damage induced upregulation of $R E C Q 1$ expression in MDA-MB-231 cells expressing mutant p53. (A) RNA was isolated from MDA-MB-231 cells that were untreated or treated with MMS $(1 \mathrm{mM})$, doxorubicin $(1 \mu \mathrm{M})$, gemcitabine $(2$ $\mu \mathrm{M})$ or camptothecin $(1 \mu \mathrm{M})$ as indicated. Fold-change in RECQ1 mRNA expression compared to untreated and normalized to GAPDH is shown. SDHA served as an additional housekeeping control. Values are average of three independent experiments and standard deviation is indicated by error bars. Statistical significance of change in RECQ1 expression compared to untreated is indicated. (B) Western Blots showing loss of p53 protein in TP53 knockout (p53_KO\#1) MDA-MB-231 clone and another clone with unchanged level of p53 (mutp53). GAPDH was used as a loading control. (C) Isogenic MDA-MB-231 cells expressing with the mutant p53 (mutp53) or knockout for p53 (p53_KO\#1) were exposed to MMS (1 mM) for $8 \mathrm{~h}$ and the fold-change in mRNA expression of RECQ1 and $\beta$-actin compared to untreated and normalized to GAPDH is shown. Statistical significance of change in RECQ1 expression compared to untreated mup53 is indicated. (D) MMS-induced enrichment of mutant 553 to RECQ1 promoter in MDA-MB-231 cells. MDA-MB-231 cells, untreated or treated with MMS (1 mM, $8 \mathrm{~h}$ ), were processed for ChIP using a p53-specific antibody. ChIP experiments with rabbit IgG served as negative control. ChIP-qPCR of immunoprecipitated DNA with primers specific for RECQ1 promoter sequence containing predicted p53 binding sites (\#1 and \#2, as shown in 3A) was performed. Fold enrichment over IgG was determined and is shown for each primer pair for the ChIP. Statistical significance of enrichment in untreated versus treatment groups is presented. Results are expressed as means \pm SEM for at least three independent experiments. 
these activities are likely to be important for its role in DNA repair [8]. Together our findings significantly expand on prior observation and indicate that transcriptional regulation of $R E C Q 1$ is potentially involved in an adaptive and/or a protective response to genotoxic stress.

Standard chemotherapy for cancer aims to produce replication stress induced DNA damage thereby promoting death preferentially in rapidly proliferating cancer
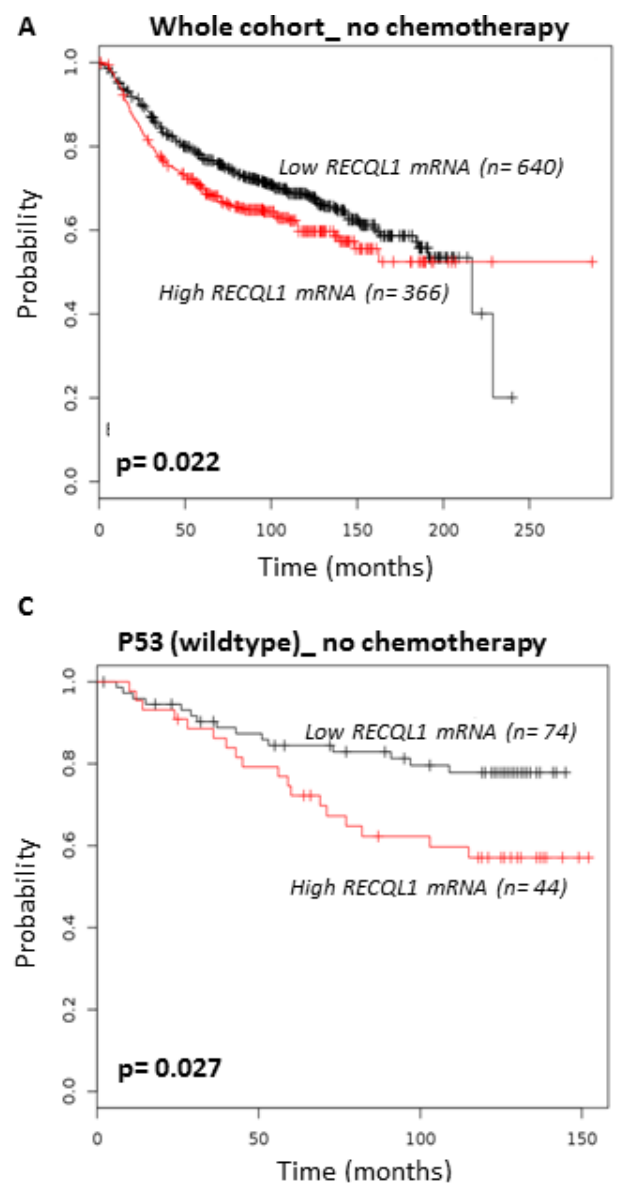

$\mathbf{E}$

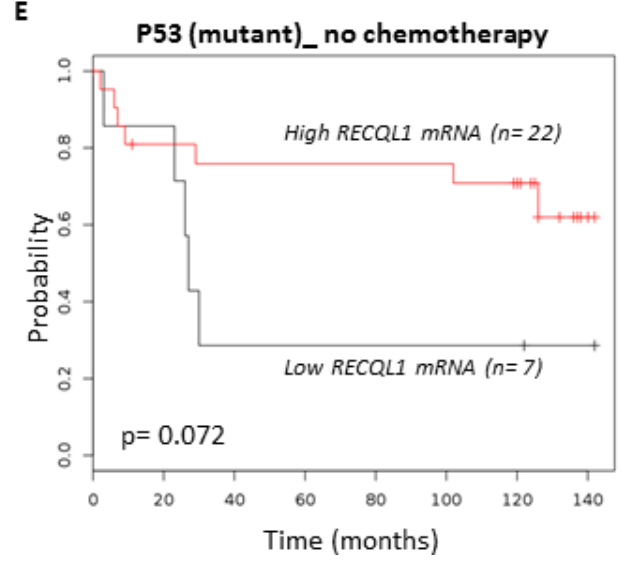

cells. However, the ability of cancer cells to recognize this damage and initiate DNA repair is an important mechanism for drug resistance and poor therapeutic efficacy [1]. Genetic variations such as mutations, copy number changes, change in mRNA expression, or single nucleotide polymorphisms in DNA repair genes may encode alterations that affect DNA repair function and, therefore, influence individual's risk of cancer development
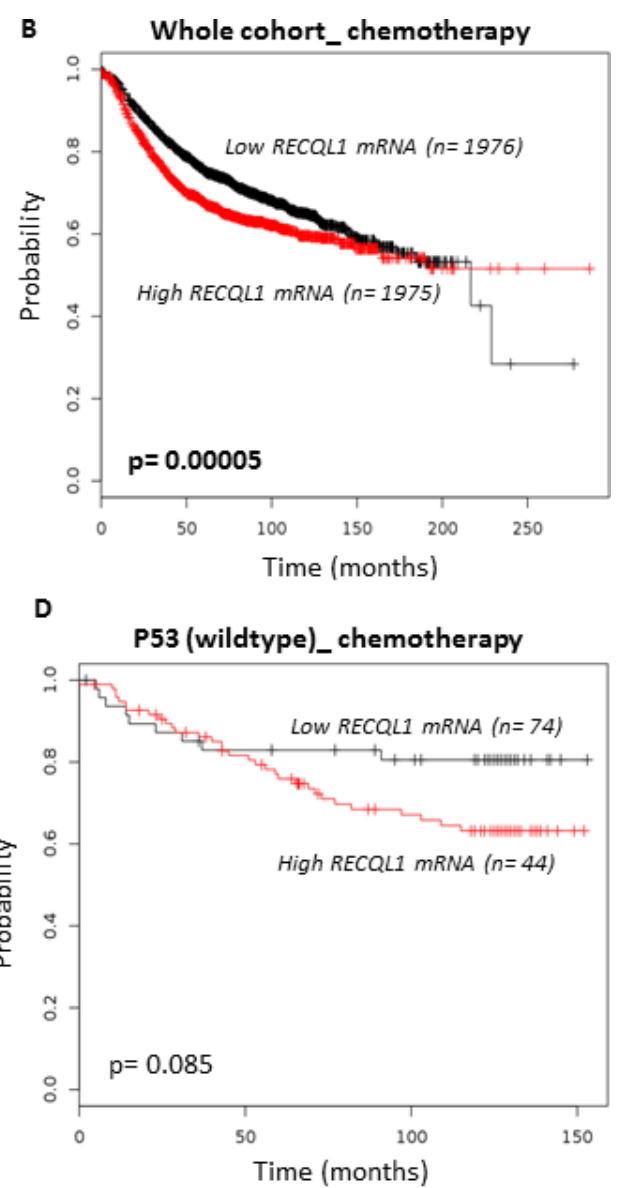

$\mathbf{F}$

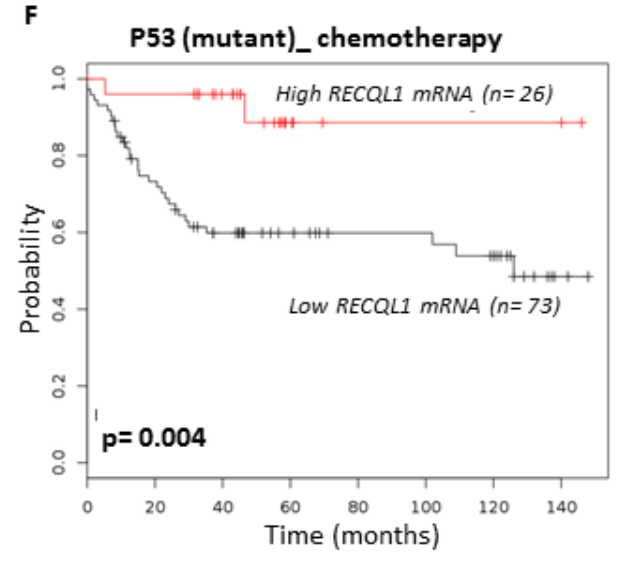

Figure 7: Clinical correlation of $R E C Q 1$ expression with p53 status for survival outcomes in breast cancer. KaplanMeier curves correlating RECQ1 mRNA expression and relapse free survival in breast cancer patients from kmplotter.com are shown. (A, B) Survival of patients, in the whole cohort of 3951 breast tumors, who received no chemotherapy (A) or did receive chemotherapy (B). (C, D) Survival curves for patients with wild-type p53 who received no chemotherapy (C) or received chemotherapy (D). (E, F) Survival curves for patients with mutant p53 who received no chemotherapy (E) or received chemotherapy (F). 
and clinical response to cytotoxic therapies [1, 2]. A polymorphism in RECQ1, A159C located in the $3^{\prime} \mathrm{UTR}$, is associated with faster tumor progression and significantly reduced survival of pancreatic adenocarcinoma patients who received gemcitabine and radiotherapy [16]. Gemcitabine, a nucleoside analog, disrupts DNA replication and interferes with the homologous recombination repair of DNA, whereas RECQ1 helicase functions are important to restore productive DNA replication [8]. It is possible that the $\mathrm{A} 159 \mathrm{C}$ variant allele confers higher $R E C Q 1$ expression, leading to a better repair of the gemcitabine-induced DNA damage and, hence, a poor clinical response. In oral squamous cell carcinoma, RECQ1 expression correlated with cisplatin resistance [59] and RECQ1-depletion significantly augmented the in vivo anticancer effects of the drug cis-platinum (II) diammine dichloride that induce inter-strand cross links in DNA to impair progression of replication forks [19]. In sporadic ER- negative breast tumors, high RECQ1 expression is associated with poor survival in patients that received anthracycline based chemotherapy; and RECQ1-depletion in breast cancer cells increased doxorubicin chemosensitivity owing to DNA double strand breaks accumulation, S-phase cell cycle arrest and apoptosis [47]. Viziteu et al. recently reported that RecQ helicases are deregulated in hematological malignancies compared to their normal counterparts and expression of RECQ1 was associated with significantly poor survival [60]. In a subsequent study, RECQ1 overexpression in multiple myeloma cells conferred resistance against the DNA alkylating drug melphalan and proteasome inhibitor bortezomib, and high expression of $R E C Q 1$ associated with poor prognosis in multiple myeloma patients treated with high dose melphalan [61]. Noteworthy, RECQ1 was significantly overexpressed in patients with deletion of chromosome $17 \mathrm{p}$ which harbors TP53, suggesting that additional factors may regulate RECQ1 expression [61]. Observed sensitivity of RECQ1depleted cells to DNA alkylating agents is consistent with a newly identified role of RECQ1 in BER [31] and a previous study where glioblastoma cells depleted of RECQ1 were found to be more sensitive to TMZ treatment [62]. Indeed, deficiency of a BER factor or imbalance in BER enzymatic steps contribute to sensitizing cells to killing effects of alkylating agents [63]. A mechanism to tolerate alkylating adducts is through the activities of translesion DNA polymerases [3]. However, unrepaired DNA adducts in such cases interfere with replication fork progression giving rise to secondary DNA damage including strand breaks [44]. RECQ1 through the repair of stalled replication forks at sites of alkylated bases and its role in BER may serve to prevent chromosome breakage upon exogenous replication stress and DNA damage induced by alkylating agents.

It is yet unclear how the missense mutations that disrupt RECQ1 enzymatic activities and altered (mostly increased) $R E C Q 1$ expression will impact breast cancer prognosis. A survey of Oncomine reveals that RECQ1 is overexpressed and amplified in many clinical cancer samples versus normal samples [27, 64]. In human cells, RECQ1 is ranked amongst the top 5-25\% most abundant proteins in Protein Abundance Database, PaxDb [65]. It can be reasoned that even a 2-fold increase in the basal level of RECQ1 protein in cells that already express it at significantly high level will likely enhance cellular capacity of DNA repair following DNA damage. Given the roles of RECQ1 in resolving replication stress, our results suggest that RECQ1 overexpression could be a marker of chemoresistance and DNA damage induced upregulation of RECQ1 would be especially important for survival of highly proliferative cancer cells. Targeted inhibition of RECQ1 expression or pharmacological inhibition of RECQ1 enzymatic activity could potentially enhance therapeutic action of anticancer drugs in p53-deficient tumor cells that are much more reliant than normal cells on pathways that resolve replication stress. Although we found that $R E C Q 1 \mathrm{mRNA}$ is induced upon DNA damage in the mutant p53-expressing MDA-MB-231 cells and this induction is lost upon targeted deletion of mutant $\mathrm{p} 53$, future studies are needed to determine if this is also observed in a panel of cell lines that express gain-offunction mutant p53 proteins. Given the established role of RECQ1 in DNA repair, it will be interesting to examine if the enhanced chemoresistance of mutant $\mathrm{p} 53$-expressing cells is mediated, in part, via induction of RECQ1.

Our demonstration that RECQ1 is a p53-responsive gene in the context of genotoxic stress suggests that a RECQ1 inhibitor may be beneficial to cancer patients who retain wild-type $\mathrm{p} 53$. Notably, and despite significant sequence conservation among the RecQ family members, specific inhibitors of WRN and BLM helicases have been developed [66, 67]. RECQ1 knockout animals are phenotypically normal [21], indicating that the RECQ1 helicase is not a general regulator of cellular proliferation and aberrant expression in cancer may be acquired on cellular transformation, suggesting targeting RECQ1 could be potentially tumor specific. The p53 tumor suppressor protein is critical in orchestrating the genomic response to stress by transcriptional regulation of genes involved in key cellular processes such as DNA repair, cell cycle arrest, senescence and apoptosis. It would be interesting to test, in future, whether concurrent p53 loss will expose the deleterious effects of genetic knockout of RECQ1 in mice which might otherwise be compensated in a p53-proficient background. Given the recent associations of RECQ1 mutations and expression with cancer susceptibility and response to therapy, it will be important to understand the roles of RECQ1 in the context of other relevant and known susceptibility genes in tumor biology.

In summary, our findings illustrate previously unknown regulation of RECQ1 expression in response to DNA damage and may be useful in understanding the clinical significance of $R E C Q 1$ expression in tumor development and therapeutic response. Identification of 
synergistic genes and pathways, and characterization of regulatory mechanisms for $R E C Q 1$ expression through the functional studies may instruct alternative therapeutic strategies.

\section{MATERIALS AND METHODS}

\section{Cell culture and DNA damage treatments}

Isogenic pair of p53-wild type (p53WT) and p53knockout (p53KO) human colon carcinoma cell lines HCT116 and RKO were provided by Dr. B. Vogelstein (The Johns Hopkins Kimmel Cancer Center, Baltimore, MD); human cervical adenocarcinoma HeLa, osteosarcoma U2OS and breast adenocarcinoma MCF7 and MDAMB-231 cell lines used in this study were purchased from American Type cell culture (ATCC); and mouse embryonic fibroblasts (MEFs) have been described [21]. Cells were maintained in Dulbecco's Modified Eagle's Medium (DMEM) containing 10\% fetal bovine serum (FBS). All cells were cultured in a humidified atmosphere containing $5 \% \mathrm{CO} 2$ at $37^{\circ} \mathrm{C}$ and routinely checked for mycoplasma contamination using a PCR based assay (Sigma, catalog no. MP0035). MMS, TMZ, FMS, doxorubicin, etoposide, camptothecin, gemcitabine, MNNG, 5-FU, and benzo[a] pyrene, were purchased from Sigma; inhibitors for ATM (Ku55933) and DNA-PK (Nu7026) were purchased from Selleck Chemicals. All stock solutions were made as recommended by the vendors and used for treatment of cells in culture.

\section{RNAi mediated knockdown of RECQ1 and p53}

On-Target plus SMARTpool small interfering RNAs (siRNAs) against RECQ1 or p53 and control siRNAs were purchased from Dharmacon. All siRNA transfections were performed by reverse transfection at a final concentration of $20 \mathrm{nM}$ using Lipofectamine RNAiMAX (Invitrogen) as instructed by the manufacturer. Stable shRNA-mediated knockdown of RECQ1 in U2OS cells was achieved using a lentiviral system [25]. Briefly, lentivirus particles were produced by cotransfecting 293T cells with the pLKO.1 lentiviral shRNA expression vector containing the RECQ1 targeting sequence (5'-GAGCTTATGTTACCAGTTA- ${ }^{\prime}$ ) or the gene encoding Luciferase (5'-ACGCTGAGTACTTCGAAATGT-3') with the packaging plasmids psPAX2 and pM2D.G; and used to transduce U2OS cells, followed by selection with puromycin $(2.5 \mu \mathrm{g} / \mathrm{ml})$.

\section{Generation of TP53 knockout MDA-MB-231 clones}

CRISPR-mediated TP53 knockout MDA-MB-231 cells were generated using an all-in-one pD1401-AD plasmid expressing the Cas9 nickase (Cas9-D10A), GFP and the 2 gRNAs targeting the TP53 exon that was common to all p53 mRNA isoforms (Supplemental figure 5). MDA-MB-231 cells were transfected with $1 \mu \mathrm{g}$ of plasmid by Nucleofection using Amaxa ${ }^{\circledR}$ Nucleofector $^{\circledR}$ Kit V (Catalog \# VCA-1003). After 48 h, transfected cells were GFP sorted and seeded at one cell per well in 96-well plates containing DMEM. Single colonies were expanded and protein was extracted using RIPA buffer. Knockout clones were genotyped by Sanger sequencing and p53 loss was confirmed by immunoblotting using an Anti-p53 antibody (DO1, Santa Cruz catalog number sc-126). gRNAs sequences targeting TP53: gRNA1- GATGGCCATGGCGCGGACGC; gRNA2GCAGTCACAGCACATGACGG.

\section{Preparation of RNA and qRT-PCR}

Total RNA was extracted from cells using Trizol (Life Technologies). The quantification of the extracted RNA was done using a NanoDrop 2000c Spectrophotometer (Thermo Scientific). For quantitative reverse transcriptionPCR (RT-qPCR) analysis, $500 \mathrm{ng}$ of total RNA was reverse transcribed using the iScript RT kit (Bio-Rad), and qPCR was performed using SYBR green (Bio-Rad) as directed by manufacturer. The glyceraldehyde-3-phosphate dehydrogenase (GAPDH) and $\beta$-Actin housekeeper genes were used as internal control. The qPCR for each RNA sample was performed in triplicate. No template control was used to rule out cross contamination of reagents and a RT minus control was used to rule out genomic DNA contamination. Statistical significance of gene expression changes in untreated versus treatment groups was confirmed using a two-tailed paired Student-t test and is presented in the figures as ${ }^{*} p<0.05 ;{ }^{\#} p<0.01 ;{ }^{* *} p<0.005 ;{ }^{\#} p<0.001$; or n. s., non-significant. The oligonucleotide sequences of the primers used for qRT-PCR are as following: RECQ1 \#1 (Forward: 5'-CAATGGCTGGAAAGGAGGTA-3'; Reverse: 5'-AATGGGCAAATGACGAGTGT-3'), RECQ1 \#2 (Forward: 5'-TGAAGCAGGCAGAGGAACTG-3'; Reverse: 5'-AGCCACAACACCTGCTACTC-3'); GAPDH (Forward: 5'-AATCCCATCACCATCTTCCA-3'; Reverse: 5'-TGGACTCCACGACGTACTCA-3'), $\beta$-actin (Forward: 5'-ACCAACTGGGACGAT ATGGAGAAGA-3'; Reverse: 5'-TACGACCAGAGGCATACAGGGACAA-3'), p21 (Forward: 5'-GACTCTCAGGGTCGAAAACG3'; Reverse: 5'-GGAT TAGGGCTTCCTCTTGG-3'), WRN (Forward: 5'-AATC TACTGAGCATTTATCTCCCA-3'; Reverse: 5'-GAGTT GGTTCTACCGTGCCA-3'), BLM (Forward: 5'-GAGT CTGCGTGCGAGGATTA-3'; Reverse: 5'-AGTGTTC TGGCTGAGTGACG-3'), RECQ4 (Forward: 5'-TCACAG TGAGGTCCCAGATT-3'; Reverse: 5'-CTGACTTCTT GGAAGGCTGA-3'), RECQ5 (Forward: 5'-GCTCAGG AAGACGGGAGAAG-3'; Reverse: 5'-AGAACAGCTT GGAGAACGGG-3'). Following two primer pairs were used for mouse: RECQ1 (Forward: 5'-GCTCTTGGCAT 
CTTGAAGCG-3'; Reverse: 5'-CTTGAGGGCTTTTGCC GAAC-3') and GAPDH (Forward: 5'-CGTGTTCCTACCC CCAATGT-3'; Reverse: 5'-GTGTAGCCCAAGATGCCC TT-3').

\section{Western blotting}

Whole-cell lysates were prepared by using radioimmunoprecipitation assay (RIPA) buffer containing protease inhibitor cocktail (Roche), and protein was quantified using DC protein assay kit (Bio-Rad). Ten microgram of total protein per lane was used for immunoblotting. The following primary antibodies were used: anti-RECQ1 (Bethyl lab; at 1:1000 dilution); anti-p53 (Santa Cruz Biotech, 1:2,500 dilution), anti-p21 (Santa Cruz Biotech, 1:500 dilution), anti-GAPDH and anti$\gamma \mathrm{H} 2 \mathrm{AX}$ (both from Cell Signaling, 1:1000 dilution).

\section{Chromatin immunoprecipitation assay}

Cells grown at a density of $1 \times 10^{7}$ per $15 \mathrm{~cm}$ diameter dish, untreated or after MMS treatment (1 $\mathrm{mM}, 8 \mathrm{~h}$ ), were used for ChIP experiments as described previously [68]. Following phenol/chloroform extraction and ethanol precipitation, sheared DNA fragments served as template in qPCR analysis. qPCR was performed using Taq Universal SYBR Green Supermix (Bio-Rad) with technical triplicates and threshold cycle numbers $\left(C_{t}\right)$ were determined with an iQ5 thermal cycler (Bio-Rad). Fold enrichment of the targeted genomic sequences were

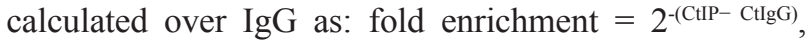
where $\mathrm{Ct}_{\mathrm{IP}}$ and $\mathrm{Ct}_{\mathrm{IgG}}$ are mean threshold cycles of PCR done in triplicates on DNA samples immunoprecipitated with anti-p53 (DO-1, Santa Cruz Biotech) or RNA POL II (CTD4H8 phospho-S5 [4H8], Abcam) antibody and control $\mathrm{IgG}$, respectively. All qPCR reactions were also checked by melt curve analyses and agarose gel electrophoresis to confirm the presence of a single specific product. Statistical significance of enrichment in ChIP in untreated versus treatment groups was confirmed using a two-tailed paired Student-t test for each genomic sequence and is presented in the figures as ${ }^{*} p<0.05 ;{ }^{*} p<0.01 ; * * p<0.005$; ${ }^{\#} p<0.001$; or n. s., non-significant. Following primers were used: primers for $-819 /+21$ region of $R E C Q 1$ promoter: \#1 (Forward: 5'-TGCTTCACAGTAGCGGAAGG-3'; Reverse: 5'- ATGTTGGAGGAAACGCCACT-3'); \#2 (Forward: 5'-CCGGTCTTCTGATCTCCCCA-3', Reverse: 5'-TTAATAACGCCCGCCCTTCC-3'); \#3 (Forward: 5'-T GCCTCTAAATGCAGGTGGC-3'; Reverse: 5'- GCA GGTCTGTCACTCAGCAG-3'), primer to the p21 promoter containing p53 RE as positive control (Forward: 5'-CTGGACTGGGCACTCTTGTC-3'; Reverse: 5'- CTC CTACCATCCCCTTCCTC-3'), and primers to GAPDH as a p53 irrelevant and non-promoter region (Forward: 5'-TACTAGCGGTTTTACGGGCG-3'; Reverse: 5'-TCG CTCTCTGCTCCTCCTGTTCGA-3').

\section{RECQ1 promoter construct}

The 5 '-region from $-819 \mathrm{bp}$ to $+21 \mathrm{bp}$, relative to the transcription start site of RECQ1 (Gene ID 5965) was cloned by PCR of genomic DNA (forward primer, 5'-AGCCAGGGACTTGTAGAGGAC-3'; reverse primer, 5'-TGGCGAAACCTGCTTCCAA-3'). The PCR product was cloned into the pGL3-Basic luciferase vector (Promega) using standard techniques. All constructs were sequence-verified.

\section{Luciferase reporter assay}

HCT116 cells, p53-proficient or p53-deficient, at $60 \%$ confluence in 6-well plates were co-transfected with $500 \mathrm{ng}$ pGL3-Basic luciferase reporter constructs containing a sequence derived from the identified p53-binding site upstream of the RECQ1 gene, (PGL3_RECQ1), wt p53 binding sites (PGL3_p53RE; a gift from Bert Vogelstein (Addgene plasmid \# 16442)[41], or empty vector and 50 ng pRL-TK Renilla reporter (Promega) by Lipofectamine 2000 as described by the manufacture's protocol. Cells were lysed $36 \mathrm{~h}$ post-transfection and luciferase activity was measured using the Dual-Luciferase Reporter Assay System (Promega) according to protocol. Firefly luciferase activities were normalized with Renilla luciferase activities to obtain the relative luciferase activity. Luciferase activity for pGL3 RECQ1 was comparable to that of pGL3 p53RE. Results are presented as normalized relative luciferase activity compared to p53-proficient HCT116 cells.

\section{Survival assay}

Cells, seeded at a density of $3 \times 10^{3}$ cells per well in 96 well plates, were either untreated or treated with DNA damaging agents in complete DMEM medium as indicated. Cell proliferation was evaluated each day by trypan blue exclusion assay and counting the viable cells using an automated cell counter (Bio-Rad TC10). Cell proliferation experiments were done in triplicates in three independent experiments.

\section{Comet assay}

The neutral comet assays were performed using a Comet Assay kit (4250-050-ESK, Trevigen) according to the manufacturer's recommendations. Cells untreated or treated with doxorubicin $(1 \mu \mathrm{M})$ or MMS $(1 \mathrm{mM})$ for $4 \mathrm{~h}$ were trypsinized and embedded on a microscope slide in agarose. Slides were incubated for $30 \mathrm{~min}$ at $4^{\circ} \mathrm{C}$ in a $\mathrm{CO}_{2}$ incubator. Cells on slides were lysed for $1 \mathrm{~h}$ at $4^{\circ} \mathrm{C}$ and the slides were then incubated in the dark for $30 \mathrm{~min}$ in cold neutral electrophoresis buffer prior to electrophoresis at $25 \mathrm{~V}$ for $25 \mathrm{~min}$. After immersion of slides for $30 \mathrm{~min}$ each in DNA precipitation solution and 70\% ethanol, DNA was visualized using SYBR Green I fluorescent staining. 
Fifty cells per sample were documented in each case. Data was analyzed using an open-source software tool, OpenComet. Results are presented as normalized \% tail moment compared to untreated control knockdown cells.

\section{RECQ1 gene expression in human breast cancers}

We initially investigated $R E C Q 1$ gene expression in the Breast Invasive Carcinoma TCGA cohort [54] using the publicly available cBioPortal for Cancer Genomics [69]. We then validated predictive significance of $R E C Q 1$ in a large cohort of 3951 breast cancers [55]. Relapse free survival was defined as the number of months from diagnosis to the occurrence of local recurrence, local lymph node $(\mathrm{LN})$ relapse or distant metastasis $(\mathrm{DM})$ relapse.

\section{Abbreviations}

All abbreviations have been defined within the manuscript.

\section{Authors' contributions}

$\mathrm{XL}$ and SP designed and conducted experiments, analyzed data; MS contributed to clinical data analysis; RC and AL contributed p53-CRISPR clones, SS supervised study design, experiments, data collection, wrote the manuscript, and all authors approved the final manuscript before submission.

\section{ACKNOWLEDGMENTS}

We thank Dr. Bert Vogelstein (Department of Pathology, Johns Hopkins University School of Medicine) for providing isogenic p53 wild-type and p53-knockout HCT116 and RKO cell lines. We thank Ms. Derrin Sodipo (supported by HUADAR program (NIA/NIH grant AG047843)) for her help with preliminary experiments during summer internship.

\section{CONFLICTS OF INTERESTS}

The authors do not have any conflicts of interest.

\section{FUNDING}

Research reported in this publication was supported by the National Institute of General Medical Sciences of the National Institutes of Health under Award Number SC1GM093999 to Sudha Sharma, and the Intramural Research Program of the National Institutes of Health, National Cancer Institute, and Center for Cancer Research to Ashish Lal. The content is solely the responsibility of the authors and does not necessarily represent the official views of the National Institutes of Health.

\section{REFERENCES}

1. Jackson SP, Helleday T. DNA REPAIR. Drugging DNA repair. Science. 2016; 352:1178-79.

2. Roos WP, Thomas AD, Kaina B. DNA damage and the balance between survival and death in cancer biology. Nat Rev Cancer. 2016; 16:20-33.

3. Christmann M, Kaina B. Transcriptional regulation of human DNA repair genes following genotoxic stress: trigger mechanisms, inducible responses and genotoxic adaptation. Nucleic Acids Res. 2013; 41:8403-20.

4. Brosh RM Jr. DNA helicases involved in DNA repair and their roles in cancer. Nat Rev Cancer. 2013; 13:542-58.

5. Croteau DL, Popuri V, Opresko PL, Bohr VA. Human RecQ helicases in DNA repair, recombination, and replication. Annu Rev Biochem. 2014; 83:519-52.

6. Larsen NB, Hickson ID. RecQ Helicases: Conserved Guardians of Genomic Integrity. Adv Exp Med Biol. 2013; 767:161-84.

7. Shamanna RA, Lu H, Croteau DL, Arora A, Agarwal D, Ball G, Aleskandarany MA, Ellis IO, Pommier Y, Madhusudan S, Bohr VA. Camptothecin targets WRN protein: mechanism and relevance in clinical breast cancer. Oncotarget. 2016; 7:13269-84. https://doi.org/10.18632/oncotarget.7906.

8. Sami F, Sharma S. Probing Genome Maintenance Functions of human RECQ1. Comput Struct Biotechnol J. 2013; 6:e201303014.

9. Cybulski C, Carrot-Zhang J, Kluźniak W, Rivera B, Kashyap A, Wokołorczyk D, Giroux S, Nadaf J, Hamel N, Zhang S, Huzarski T, Gronwald J, Byrski T, et al. Germline RECQL mutations are associated with breast cancer susceptibility. Nat Genet. 2015; 47:643-46.

10. Sun J, Wang Y, Xia Y, Xu Y, Ouyang T, Li J, Wang T, Fan Z, Fan T, Lin B, Lou H, Xie Y. Mutations in RECQL Gene Are Associated with Predisposition to Breast Cancer. PLoS Genet. 2015; 11:e1005228.

11. Kawabe T, Tsuyama N, Kitao S, Nishikawa K, Shimamoto A, Shiratori M, Matsumoto T, Anno K, Sato T, Mitsui Y, Seki M, Enomoto T, Goto M, et al. Differential regulation of human RecQ family helicases in cell transformation and cell cycle. Oncogene. 2000; 19:4764-72.

12. Futami K, Kumagai E, Makino H, Sato A, Takagi M, Shimamoto A, Furuichi Y. Anticancer activity of RecQL1 helicase siRNA in mouse xenograft models. Cancer Sci. 2008; 99:1227-36.

13. Futami K, Kumagai E, Makino H, Goto H, Takagi M, Shimamoto A, Furuichi Y. Induction of mitotic cell death in cancer cells by small interference RNA suppressing the expression of RecQL1 helicase. Cancer Sci. 2008; 99:71-80.

14. Li XL, Lu X, Parvathaneni S, Bilke S, Zhang H, Thangavel S, Vindigni A, Hara T, Zhu Y, Meltzer PS, Lal A, Sharma S. Identification of RECQ1-regulated transcriptome uncovers a role of RECQ1 in regulation of cancer cell migration and invasion. Cell Cycle. 2014; 13:2431-45. 
15. Sharma S. An appraisal of RECQ1 expression in cancer progression. Front Genet. 2014; 5:426.

16. Li D, Frazier M, Evans DB, Hess KR, Crane CH, Jiao L, Abbruzzese JL. Single nucleotide polymorphisms of RecQ1, RAD54L, and ATM genes are associated with reduced survival of pancreatic cancer. J Clin Oncol. 2006; 24:1720-28.

17. Futami K, Furuichi Y. RECQL1 and WRN DNA repair helicases: potential therapeutic targets and proliferative markers against cancers. Front Genet. 2015; 5:441.

18. Futami K, Ogasawara S, Goto H, Yano H, Furuichi Y. RecQL1 DNA repair helicase: A potential tumor marker and therapeutic target against hepatocellular carcinoma. Int J Mol Med. 2010; 25:537-45.

19. Arai A, Chano T, Futami K, Furuichi Y, Ikebuchi K, Inui T, Tameno H, Ochi Y, Shimada T, Hisa Y, Okabe H. RECQL1 and WRN proteins are potential therapeutic targets in head and neck squamous cell carcinoma. Cancer Res. 2011; 71:4598-607.

20. Sanada S, Futami K, Terada A, Yonemoto K, Ogasawara S, Akiba J, Yasumoto M, Sumi A, Ushijima K, Kamura T, Furuichi Y, Yano H. RECQL1 DNA repair helicase: a potential therapeutic target and a proliferative marker against ovarian cancer. PLoS One. 2013; 8:e72820.

21. Sharma S, Stumpo DJ, Balajee AS, Bock CB, Lansdorp PM, Brosh RM Jr, Blackshear PJ. RECQL, a member of the RecQ family of DNA helicases, suppresses chromosomal instability. Mol Cell Biol. 2007; 27:1784-94.

22. Sharma S, Brosh RM Jr. Human RECQ1 is a DNA damage responsive protein required for genotoxic stress resistance and suppression of sister chromatid exchanges. PLoS One. 2007; 2:e1297.

23. Sami F, Lu X, Parvathaneni S, Roy R, Gary RK, Sharma S. RECQ1 interacts with FEN-1 and promotes binding of FEN1 to telomeric chromatin. Biochem J. 2015; 468:227-44.

24. Popuri V, Hsu J, Khadka P, Horvath K, Liu Y, Croteau DL, Bohr VA. Human RECQL1 participates in telomere maintenance. Nucleic Acids Res. 2014; 42:5671-88.

25. Berti M, Ray Chaudhuri A, Thangavel S, Gomathinayagam S, Kenig S, Vujanovic M, Odreman F, Glatter T, Graziano S, Mendoza-Maldonado R, Marino F, Lucic B, Biasin V, et al. Human RECQ1 promotes restart of replication forks reversed by DNA topoisomerase I inhibition. Nat Struct Mol Biol. 2013; 20:347-54.

26. Popuri V, Croteau DL, Brosh RM Jr, Bohr VA. RECQ1 is required for cellular resistance to replication stress and catalyzes strand exchange on stalled replication fork structures. Cell Cycle. 2012; 11:4252-65.

27. Lu X, Parvathaneni S, Hara T, Lal A, Sharma S. Replication stress induces specific enrichment of RECQ1 at common fragile sites FRA3B and FRA16D. Mol Cancer. 2013; 12:29.
28. Parvathaneni S, Stortchevoi A, Sommers JA, Brosh RM Jr, Sharma S. Human RECQ1 interacts with Ku70/80 and modulates DNA end-joining of double-strand breaks. PLoS One. 2013; 8:e62481.

29. Sharma S, Phatak P, Stortchevoi A, Jasin M, Larocque JR. RECQ1 plays a distinct role in cellular response to oxidative DNA damage. DNA Repair (Amst). 2012; 11:537-49.

30. Khan I, Suhasini AN, Banerjee T, Sommers JA, Kaplan DL, Kuper J, Kisker C, Brosh RM Jr. Impact of age-associated cyclopurine lesions on DNA repair helicases. PLoS One. 2014; 9:e113293.

31. Woodrick J, Gupta S, Camacho S, Parvathaneni S, Choudhury S, Cheema A, Bai Y, Khatkar P, Erkizan HV, Sami F, Su Y, Schärer OD, Sharma S, Roy R. A new subpathway of long-patch base excision repair involving 5' gap formation. EMBO J. 2017; 36:1605-22.

32. Efeyan A, Serrano M. p53: guardian of the genome and policeman of the oncogenes. Cell Cycle. 2007; 6:1006-10.

33. Khoronenkova SV, Dianov GL. ATM prevents DSB formation by coordinating SSB repair and cell cycle progression. Proc Natl Acad Sci USA. 2015; 112:3997-4002.

34. Kharbanda S, Pandey P, Jin S, Inoue S, Bharti A, Yuan ZM, Weichselbaum R, Weaver D, Kufe D. Functional interaction between DNA-PK and c-Abl in response to DNA damage. Nature. 1997; 386:732-35.

35. Lakin ND, Jackson SP. Regulation of p53 in response to DNA damage. Oncogene. 1999; 18:7644-55.

36. Macleod KF, Sherry N, Hannon G, Beach D, Tokino T, Kinzler $\mathrm{K}$, Vogelstein B, Jacks T. p53-dependent and independent expression of p21 during cell growth, differentiation, and DNA damage. Genes Dev. 1995; 9:935-44.

37. Sengupta S, Shimamoto A, Koshiji M, Pedeux R, Rusin M, Spillare EA, Shen JC, Huang LE, Lindor NM, Furuichi Y, Harris CC. Tumor suppressor p53 represses transcription of RECQ4 helicase. Oncogene. 2005; 24:1738-48.

38. el-Deiry WS, Kern SE, Pietenpol JA, Kinzler KW, Vogelstein B. Definition of a consensus binding site for p53. Nat Genet. 1992; 1:45-49.

39. Menendez D, Inga A, Resnick MA. The expanding universe of p53 targets. Nat Rev Cancer. 2009; 9:724-37.

40. Farré D, Roset R, Huerta M, Adsuara JE, Roselló L, Albà MM, Messeguer X. Identification of patterns in biological sequences at the ALGGEN server: PROMO and MALGEN. Nucleic Acids Res. 2003; 31:3651-53.

41. el-Deiry WS, Tokino T, Velculescu VE, Levy DB, Parsons R, Trent JM, Lin D, Mercer WE, Kinzler KW, Vogelstein B. WAF1, a potential mediator of p53 tumor suppression. Cell. 1993; 75:817-25.

42. Menendez D, Nguyen TA, Freudenberg JM, Mathew VJ, Anderson CW, Jothi R, Resnick MA. Diverse stresses dramatically alter genome-wide p53 binding and transactivation landscape in human cancer cells. Nucleic Acids Res. 2013; 41:7286-301. 
43. Bunz F, Hwang PM, Torrance C, Waldman T, Zhang Y, Dillehay L, Williams J, Lengauer C, Kinzler KW, Vogelstein B. Disruption of p53 in human cancer cells alters the responses to therapeutic agents. J Clin Invest. 1999; 104:263-69.

44. Lundin $\mathrm{C}$, North $\mathrm{M}$, Erixon $\mathrm{K}$, Walters $\mathrm{K}$, Jenssen $\mathrm{D}$, Goldman AS, Helleday T. Methyl methanesulfonate (MMS) produces heat-labile DNA damage but no detectable in vivo DNA double-strand breaks. Nucleic Acids Res. 2005; 33:3799-811.

45. Groth P, Ausländer S, Majumder MM, Schultz N, Johansson F, Petermann E, Helleday T. Methylated DNA causes a physical block to replication forks independently of damage signalling, $\mathrm{O}(6)$-methylguanine or DNA single-strand breaks and results in DNA damage. J Mol Biol. 2010; 402:70-82.

46. Kurz EU, Douglas P, Lees-Miller SP. Doxorubicin activates ATM-dependent phosphorylation of multiple downstream targets in part through the generation of reactive oxygen species. J Biol Chem. 2004; 279:53272-81.

47. Arora A, Parvathaneni S, Aleskandarany MA, Agarwal D, Ali R, Abdel-Fatah T, Green AR, Ball GR, Rakha EA, Ellis IO, Sharma S, Madhusudan S. Clinicopathological and Functional Significance of RECQL1 Helicase in Sporadic Breast Cancers. Mol Cancer Ther. 2017; 16:239-50.

48. Nikolova T, Roos WP, Kramer OH, Strik HM, Kaina B. Chloroethylating nitrosoureas in cancer therapy: DNA damage, repair and cell death signaling. Biochim Biophys Acta. 2017; 1868:29-39.

49. Garige M, Sharma S. Cellular deficiency of Werner syndrome protein or RECQ1 promotes genotoxic potential of hydroquinone and benzo[a]pyrene exposure. Int J Toxicol. 2014; 33:373-81.

50. Muller PA, Vousden KH. Mutant p53 in cancer: new functions and therapeutic opportunities. Cancer Cell. 2014; 25:304-17.

51. Strano S, Dell'Orso S, Di Agostino S, Fontemaggi G, Sacchi A, Blandino G. Mutant p53: an oncogenic transcription factor. Oncogene. 2007; 26:2212-19.

52. Walerych D, Napoli M, Collavin L, Del Sal G. The rebel angel: mutant p53 as the driving oncogene in breast cancer. Carcinogenesis. 2012; 33:2007-17.

53. Zhu J, Sammons MA, Donahue G, Dou Z, Vedadi M, Getlik M, Barsyte-Lovejoy D, Al-Awar R, Katona BW, Shilatifard A, Huang J, Hua X, Arrowsmith CH, Berger SL. Gain-offunction p53 mutants co-opt chromatin pathways to drive cancer growth. Nature. 2015; 525:206-11.

54. Ciriello G, Gatza ML, Beck AH, Wilkerson MD, Rhie SK, Pastore A, Zhang H, McLellan M, Yau C, Kandoth C, Bowlby R, Shen H, Hayat S, et al, and TCGA Research Network. Comprehensive Molecular Portraits of Invasive Lobular Breast Cancer. Cell. 2015; 163:506-19.

55. Györffy B, Lanczky A, Eklund AC, Denkert C, Budczies J, Li Q, Szallasi Z. An online survival analysis tool to rapidly assess the effect of 22,277 genes on breast cancer prognosis using microarray data of 1,809 patients. Breast Cancer Res Treat. 2010; 123:725-31.

56. Boisvert FM, Lamond AI. p53-Dependent subcellular proteome localization following DNA damage. Proteomics. 2010; 10:4087-97.

57. Sharma S, Brosh RM Jr. Unique and important consequences of RECQ1 deficiency in mammalian cells. Cell Cycle. 2008; 7:989-1000.

58. Sharma S, Sommers JA, Choudhary S, Faulkner JK, Cui S, Andreoli L, Muzzolini L, Vindigni A, Brosh RM Jr. Biochemical analysis of the DNA unwinding and strand annealing activities catalyzed by human RECQ1. J Biol Chem. 2005; 280:28072-84.

59. Zhang P, Zhang Z, Zhou X, Qiu W, Chen F, Chen W. Identification of genes associated with cisplatin resistance in human oral squamous cell carcinoma cell line. BMC Cancer. 2006; 6:224.

60. Viziteu E, Kassambara A, Pasero P, Klein B, Moreaux J. RECQ helicases are deregulated in hematological malignancies in association with a prognostic value. Biomark Res. 2016; 4:3.

61. Viziteu E, Klein B, Basbous J, Lin YL, Hirtz C, Gourzones C, Tiers L, Bruyer A, Vincent L, Grandmougin C, Seckinger A, Goldschmidt H, Constantinou A, et al. RECQ1 helicase is involved in replication stress survival and drug resistance in multiple myeloma. Leukemia. 2017 Mar 10. doi: 10.1038/ leu.2017.54. [Epub ahead of print]

62. Mendoza-Maldonado R, Faoro V, Bajpai S, Berti M, Odreman F, Vindigni M, Ius T, Ghasemian A, Bonin S, Skrap M, Stanta G, Vindigni A. The human RECQ1 helicase is highly expressed in glioblastoma and plays an important role in tumor cell proliferation. Mol Cancer. 2011; 10:83.

63. Kondo N, Takahashi A, Ono K, Ohnishi T. DNA Damage Induced by Alkylating Agents and Repair Pathways. J Nucleic Acids. 2010; 2010:543531.

64. Rhodes DR, Yu J, Shanker K, Deshpande N, Varambally R, Ghosh D, Barrette T, Pandey A, Chinnaiyan AM. ONCOMINE: a cancer microarray database and integrated data-mining platform. Neoplasia. 2004; 6:1-6.

65. Wang M, Weiss M, Simonovic M, Haertinger G, Schrimpf SP, Hengartner MO, von Mering C. PaxDb, a database of protein abundance averages across all three domains of life. Mol Cell Proteomics. 2012; 11:492-500.

66. Aggarwal M, Sommers JA, Shoemaker RH, Brosh RM Jr. Inhibition of helicase activity by a small molecule impairs Werner syndrome helicase (WRN) function in the cellular response to DNA damage or replication stress. Proc Natl Acad Sci USA. 2011; 108:1525-30.

67. Rosenthal AS, Dexheimer TS, Gileadi O, Nguyen GH, Chu WK, Hickson ID, Jadhav A, Simeonov A, Maloney DJ. Synthesis and SAR studies of 5-(pyridin-4-yl)-1,3,4thiadiazol-2-amine derivatives as potent inhibitors of Bloom helicase. Bioorg Med Chem Lett. 2013; 23:5660-66. 
68. Lu X, Parvathaneni S, Li XL, Lal A, Sharma S. Transcriptome guided identification of novel functions of RECQ1 helicase. Methods. 2016; 108:111-17.

69. Cerami E, Gao J, Dogrusoz U, Gross BE, Sumer SO, Aksoy BA, Jacobsen A, Byrne CJ, Heuer ML, Larsson E, Antipin
Y, Reva B, Goldberg AP, et al. The cBio cancer genomics portal: an open platform for exploring multidimensional cancer genomics data. Cancer Discov. 2012; 2:401-04. 